Draft version August 31, 2021

Typeset using LATEX twocolumn style in AASTeX63

\title{
Transiting Exoplanet Monitoring Project (TEMP). VI. The Homogeneous Refinement of System Parameters for 39 Transiting Hot Jupiters with 127 New Light Curves
}

\author{
Xian-Yu Wang (iD, ${ }^{2,3}$ Yong-Hao Wang (D), ${ }^{1}$ Songhu Wang (D), ${ }^{4}$ Zhen-Yu Wu (D), ${ }^{2,3}$ Malena Rice (D), ${ }^{5}$ Xu Zhou, ${ }^{2}$ \\ Tobias C. Hinse (D), ${ }^{6,7}$ Hui-Gen Liu (D) ${ }^{8}$ Bo Ma (D), ${ }^{1}$ Xiyan Peng, ${ }^{2}$ Hui Zhang (DD, ${ }^{8,9}$ Cong Yu (D), ${ }^{1}$ Ji-Lin Zhou (D), ${ }^{8}$ \\ And Gregory Laughlin (D) 5

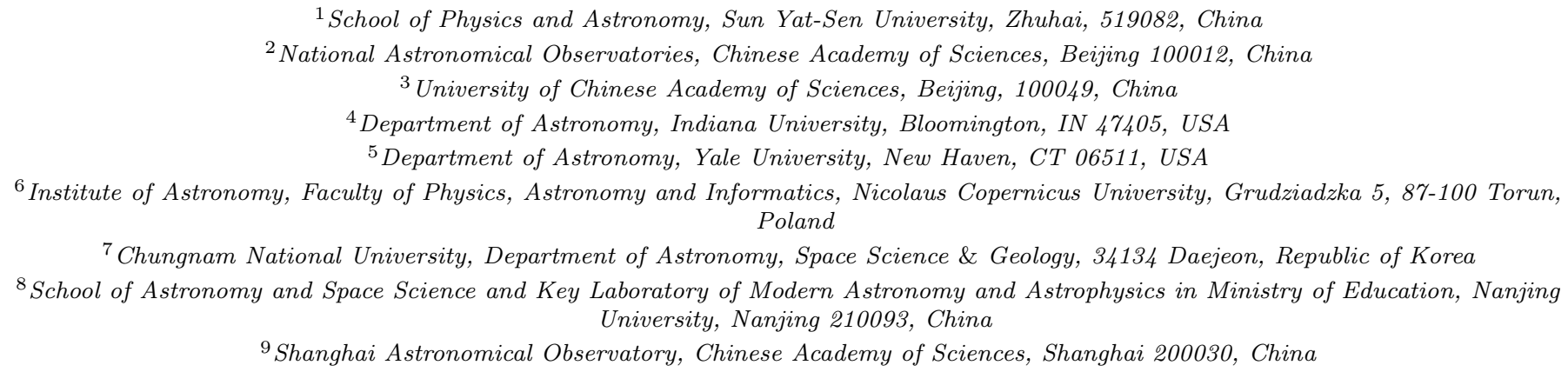

\begin{abstract}
We present 127 new transit light curves for 39 hot Jupiter systems, obtained over the span of five years by two ground-based telescopes. A homogeneous analysis of these newly collected light curves together with archived spectroscopic, photometric, and Doppler velocimetric data using EXOFASTv2 leads to a significant improvement in the physical and orbital parameters of each system. All of our stellar radii are constrained to accuracies of better than $3 \%$. The planetary radii for 37 of our 39 targets are determined to accuracies of better than $5 \%$. Compared to our results, the literature eccentricities are preferentially overestimated due to the Lucy-Sweeney bias. Our new photometric observations therefore allow for significant improvement in the orbital ephemerides of each system. Our correction of the future transit window amounts to a change exceeding $10 \mathrm{~min}$ for ten targets at the time of JWST's launch, including a $72 \mathrm{~min}$ change for WASP-56. The measured transit mid-times for both literature light curves and our new photometry show no significant deviations from the updated linear ephemerides, ruling out in each system the presence of companion planets with masses greater than $0.39-5.0 \mathrm{M}_{\oplus}, 1.23-14.36 \mathrm{M}_{\oplus}, 1.65-21.18 \mathrm{M}_{\oplus}$, and $0.69-6.75 \mathrm{M}_{\oplus}$ near the 1:2, 2:3, 3:2, and 2:1 resonances with the hot Jupiters, respectively, at a confidence level of $\pm 1 \sigma$. The absence of resonant companion planets in the hot Jupiter systems is inconsistent with the conventional expectation from disk migration.
\end{abstract}

Keywords: planets and satellites: fundamental parameters — stars: fundamental parameters techniques: photometric

\section{INTRODUCTION}

Xian-Yu Wang

xianyu_wang@nao.cas.cn

Yong-Hao Wang

wangyhao5@mail.sysu.edu.cn
Thousands of exoplanets have been detected to date; among them, transiting hot Jupiters are the most observationally accessible class of exoplanets and hence provide the highest signal-to-noise data (see, e.g., new discoveries from the Transiting Exoplanet Survey Satellite (TESS): Wang et al. 2019; Cañas et al. 2019; Jones et al. 2019; Addison et al. 2020; Brahm et al. 2020; Davis 
et al. 2020; Jordán et al. 2020). These transiting "hot Jupiters" provide us with unique laboratories to test theories of exoplanet formation and evolution with relatively high precision over short time scales.

The frequency of a planet's transits encodes the orbital period, and the depth of these transits encodes the planetary radius, assuming we know the stellar radius. A combination of photometric radius measurements and Doppler mass determinations enables us to measure the bulk density of exoplanets, helping us to understand what they are made of and how they formed. Some hot Jupiters have unusually low densities by comparison with the expected values from structural models (Laughlin et al. 2011). The dominant mechanism that slows down their convective cooling is still poorly known (Thorngren \& Fortney 2018).

Moreover, transiting hot Jupiters around bright stars enable the study of their stellar obliquities via the Rossiter-Mclaughlin effect (Holt 1893; Schlesinger 1910; Rossiter 1924; McLaughlin 1924; Queloz et al. 2000; Hébrard et al. 2008; Albrecht et al. 2012; Zhou et al. 2016; Wang et al. 2018a; Addison et al. 2018; Winn \& Fabrycky 2015 and references within), planetary atmospheres using transmission spectroscopy (Charbonneau et al. 2002; Kreidberg et al. 2014; Deming \& Seager 2017 and references within), and thermal emission through secondary eclipses (Charbonneau et al. 2005; Deming et al. 2005) and phase variations (Borucki et al. 2009; Knutson et al. 2012). Transiting hot Jupiters around bright stars also present the opportunity to measure planetary oblateness (Carter \& Winn 2010), rotation rates (Seager \& Hui 2002), orbital variations (e.g. transit timing variations (TTVs), Holman et al. 2006; Millholland et al. 2016; Wu et al. 2018; orbital decay, Schlaufman \& Winn 2013; Penev et al. 2018, orbital procession, Bouma et al. 2019), and planetary system architectures (Becker et al. 2015; Cañas et al. 2019; Huang et al. 2020). These observational properties afford a unique opportunity for us to understand the underlying physics of tidal and perturbation theory, as well as planet formation mechanisms.

Although the number and variety of exoplanets have been dramatically expanded by space missions (CoRoT, Auvergne et al. 2009; Kepler, Borucki et al. 2010; K2, Howell et al. 2014; TESS, Ricker et al. 2015), most of the currently known hot Jupiters were discovered by ground-based transit surveys, such as SuperWASP (Pollacco et al. 2006), HATNet (Bakos et al. 2004), HATSouth (Bakos et al. 2013), OGLE (Udalski et al. 2002), TRES (Alonso et al. 2004), QES (Alsubai et al. 2013), CSTAR (Wang et al. 2014a), KELT (Pepper et al. 2007), XO (McCullough et al. 2005), MASCARA (Talens et al.
2017), MEarth (Irwin et al. 2009), and NGTS (Wheatley et al. 2018).

Some hot Jupiters that are discovered by groundbased transit surveys receive only a handful of photometric follow-up observations. We therefore initiated a geographically dispersed Transiting Exoplanet Monitoring Project (TEMP, Wang et al. 2018b) by leveraging abundant 1m-class telescopes, to which we have access, to gather long-term, high-quality photometry for currently known hot Jupiters. Considering the target visibility, telescope size, and photometric precision that we usually achieve, we set the following three criteria for target selection: 1). Targets are above a declination of $\left.-20^{\circ} ; 2\right)$. The magnitudes of targets are between $V=7.5$ and $V=14.5$; and 3 ). The planetary transit has depth $>0.005$ mag. TEMP offers a powerful tool for us to achieve several goals. First, we can refine the orbital and physical parameters of the known transiting hot Jupiters discovered with ground-based photometric surveys. Second, we can identify statistically significant TTVs, which can be caused not only by planet-planet interactions (Wang et al. 2017), but also by tidal dissipation, as well as apsidal precession caused by the stellar quadrupole moment, general relativity, and long-period planetary/stellar companions (Pál \& Kocsis 2008).

In this paper, we present 127 new photometric light curves for 39 transiting hot Jupiters. By jointly analyzing our light curves together with archived photometric and Doppler velocimetric data, as well as stellar information, we report refined system parameters for these targets.

This paper is organized as follows. In Section 2, we describe the new photometric observations and their reduction. Section 3 details the technique we used to estimate the system parameters. Section 4 discusses our results and their implications.

\section{OBSERVATIONS AND DATA REDUCTION}

We collected 127 transits for 39 hot Jupiters with the $60 / 90 \mathrm{~cm}$ Schmidt telescope and the $60 \mathrm{~cm}$ telescope at the Xinglong Station of the National Astronomical Observatory of China (NAOC), which lies about 75 miles $(120 \mathrm{~km})$ to the northeast of Beijing. A total of 23,519 exposures ( 454 hours, spread across 102 nights) were collected between Nov 11, 2013 and May 8, 2018. Figure 1 shows the distribution of TEMP targets across the sky. The allocation of transit observations reported in this work is displayed for each target in Figure 2, described in detail in the following sections.

\subsection{Xinglong 60/90 cm Schmidt Telescope}

We observed 49 transits of 24 planets in $R$-band using the Xinglong 60/90 cm Schmidt telescope. The telescope 


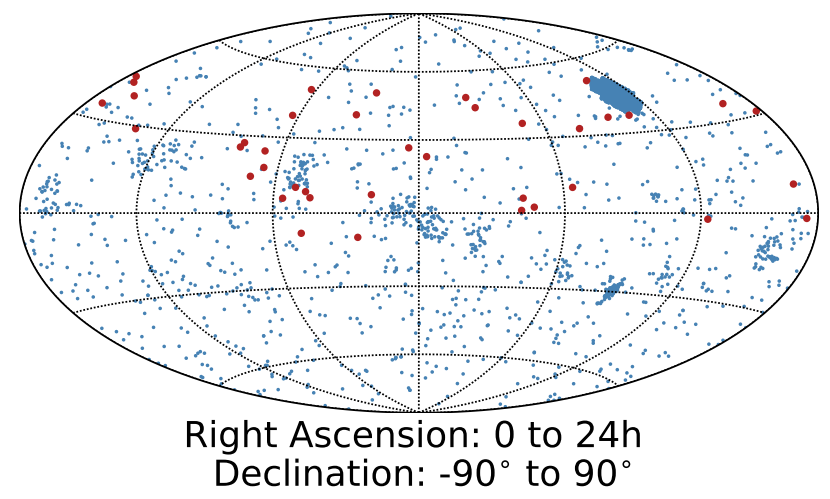

Figure 1. The distribution of observed TEMP targets (red dots). We collected 127 transit light curves for 39 hot Jupiter systems between Nov 11, 2013 and May 08, 2018. The brightest target in our sample is WASP-38 $(V=9.48)$ and the faintest one is HAT-P-53 $(V=13.73)$. All other currently known exoplanets are displayed in blue.

is equipped with a $4 \mathrm{~K} \times 4 \mathrm{~K} \mathrm{CCD}$, which gives a pixel scale of $1.38^{\prime \prime}$ pixel $^{-1}$ and a field of view of $94^{\prime} \times 94^{\prime}$. A $512 \times 512$ pixel $\left(\sim 12^{\prime} \times 12^{\prime}\right)$ subframe was used to significantly reduce the readout time from 1.5 minutes to 4 seconds.

More details about this telescope and instrument are given by Zhou et al. (1999, 2001).

\subsection{Xinglong $60 \mathrm{~cm}$ Telescope}

We acquired 78 light curves for 33 planets in $R$ band with the Xinglong $60 \mathrm{~cm}$ Telescope. Because of frequent equipment updates and tests, the Xinglong $60 \mathrm{~cm}$ telescope has a complicated history of CCD use. Observations taken between Jan 17, 2014 and Oct 212014 were conducted with a $512 \times 512 \mathrm{CCD}$, which covers a field of view of $17^{\prime} \times 17^{\prime}$ with a plate scale of $1.95^{\prime \prime}$ pixel $^{-1}$. Observations collected during May 05 to Oct 22, 2015 or during Jun 18, 2016 to Feb 14, 2018 were performed with a $1 \mathrm{~K} \times 1 \mathrm{~K} \mathrm{CCD}$, which covers a field of view of $17^{\prime} \times 17^{\prime}$ with a plate scale of $0.99^{\prime \prime}$ pixel $^{-1}$. Observations made between Oct 23, 2015 and May 16, 2016 were obtained with a $2 \mathrm{~K} \times 2 \mathrm{~K} \mathrm{CCD}$, which covers a field of view of $36^{\prime} \times 36^{\prime}$ with a plate scale of $1.06^{\prime \prime}$ pixel $^{-1}$.

The time of each observation taken by both telescopes was automatically set to the precise current time using the GPS function. The beginning time of each exposure was recorded in the frame header using the UTC time standard, which was then converted to $\mathrm{BJD}_{\mathrm{TDB}}$ as described in Eastman et al. (2010). To avoid non-linear effects of the CCD, the defocusing technique, which increases the duty cycle of observations to reduce the Poisson and the scintillation noise (Southworth et al. 2009), was used for bright stars. The data were reduced fol- lowing a standard photometric procedure described in Wang X. et al. (2018), Wang et al. (2018a,b), Wang Y et al. (2017), and Wang Y. et al. (2019). We first applied standard bias and flat-field corrections to all frames, then performed aperture photometry using SExtractor (Bertin \& Arnouts 1996). We identified the best aperture for both the target and reference stars as the one that minimized the root mean square (RMS) of the final differential light curves, which were obtained by comparing our target with several reference stars in the field. Highly discrepant points and/or linear trends presented in these light curves were removed. Furthermore, we quantified the quality of each light curve by its photometric noise rate (pnr; Fulton et al. 2011), which is defined as

$$
p n r=\frac{\mathrm{rms}}{\sqrt{\Gamma}}
$$

where the rms is the root mean square of the fitting residuals and $\Gamma$ is the median number of exposures per minute. Then, using the K-means clustering method (MacQueen 1967), the light curves were divided into three groups: golden, mediocre, and bad. The results of the classification show that there are four bad light curves, including HAT-P-37 on May 31 2017; WASP-36 on Mar 02 2016; and WASP-37 on Mar 262017 and on Apr 20 2018. We present these bad light curves in Table 1 but did not include them in the light curve fit. A summary of the observations is provided in Table 1 . The final light curves are presented in Table 2 and shown in Figure 3.

\section{LIGHT CURVE ANALYSIS}

\subsection{Planetary System Parameters from Global Analysis}

To refine the system parameters for our observed hot Jupiters, we used EXOFASTv2 (Eastman et al. 2013; Eastman 2017; Eastman et al. 2019) to simultaneously fit all published Doppler velocimetric and photometric data together with our newly collected light curves for each system. EXOFASTv2 enables simultaneous modeling of multi-band transits and multi-set radial velocities by combining the AMOEBA symplectic solver with a differential evolution Markov chain Monte Carlo (DEMCMC; Ter braak 2006) algorithm.

For each system, we adopted Gaussian priors from the Exoplanet Orbit Database ${ }^{1}$ for $T_{\text {eff }},[\mathrm{Fe} / \mathrm{H}]$, transit mid-times $\left(T_{0}\right)$, and orbital period $(P)$. We imposed priors on the quadratic limb-darkening coefficients from Claret (2018) based on $T_{\text {eff }},[\mathrm{Fe} / \mathrm{H}]$, and filter wave-

\footnotetext{
${ }^{1}$ http://exoplanets.org/
} 


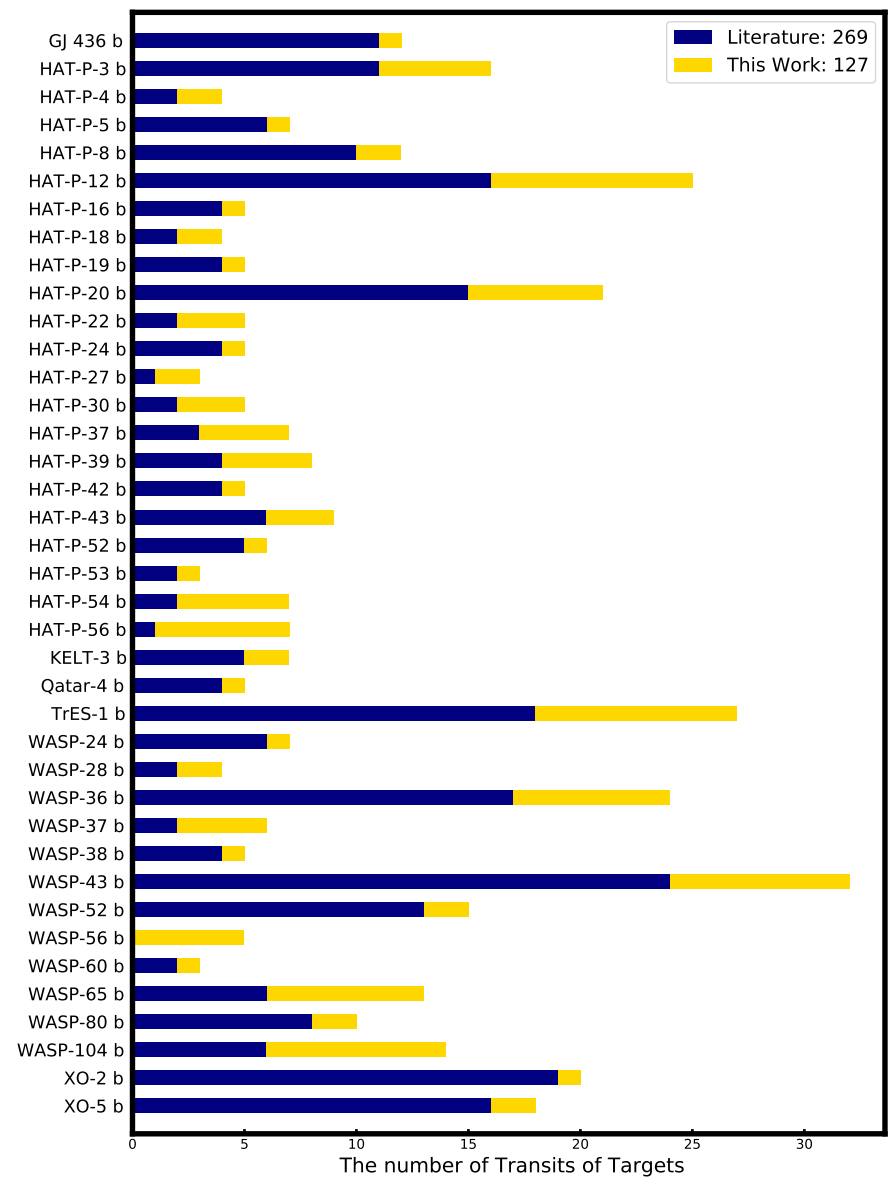

Figure 2. The number of transit light curves we used in this study (396 in total). The blue bar represents the number of archived photometric transits for each target (269 in total). The yellow bar represents the number of transits that we collected (127 in total).

length. We applied the Gaussian prior on stellar parallax to the corrected Gaia DR2 parallax (Gaia Collaboration et al. 2018; Stassun \& Torres 2018). We constrained the $V$-band extinction $\left(A_{V}\right)$ for each system by using the galactic dust maps presented in Schlafly \& Finkbeiner (2011). We also used stellar isochrones from the MESA Isochrones \& Stellar Tracks (MIST) catalog and constructed spectral energy distributions (SEDs) from a series of photometric catalogs ${ }^{2}$ to estimate the host stellar parameters.

${ }^{2}$ Galex (Bianchi et al. 2011), Tycho-2 (Høg et al. 2000), UCAC4 (Zacharias et al. 2012), APASS (Henden et al. 2016), 2MASS (Cutri et al. 2003), WISE (Cutri \& Et 2013), Gaia (Gaia Collaboration et al. 2016), the Kepler INT Survey (Greiss et al. 2012), the UBV Photoelectric Catalog (Mermilliod 1994), and the Stroemgren-Crawford uvby photometry catalog (Paunzen 2015).
All the fittings were converged by the Gelman-Rubin diagnostic ( $<1.01$, Gelman \& Rubin 1992). All of our new light curves and phased-folded Doppler velocities are compared in Figure 3 and Figure 4 to the respective best-fitting models.

\subsection{Transit Mid-Times from Individual Fitting}

Following the routine described in Wang X. et al. (2018), we used the JKTEBOP tool (Southworth et al. $2004 \mathrm{a}, \mathrm{b})$ to fit each observed transit and to subsequently derive the associated transit mid-times. For each target, we fixed all global parameters to the results derived from the global fitting in Section 3.1. The transit mid-time $\left(T_{0}\right)$ and baseline flux $\left(F_{0}\right)$ are the only two free parameters that we fit.

The best-fitting transit mid-times for each target were obtained by using the Levenberg-Marquardt non-linear least-squares fitting algorithm (Press et al. 1992). To obtain reliable uncertainties for the transit mid-times, the bootstrapping method, Monte Carlo simulations, and the residual-shift method were each employed independently. We chose the largest uncertainty derived from the above three methods for a conservative estimate.

The resulting transit mid-times for each target are reported in Table 3. Based on the transit mid-times, we derived the updated linear orbital ephemerides for each target using the weighted least-squares fitting algorithm. We chose the epoch in our observations that corresponds to the minimum covariance with the orbital period as the reference epoch (Mallonn et al. 2019). The deviations of transit mid-times for each target from the updated linear orbital ephemerides determined in this work are plotted in Figure 5.

\section{RESULTS AND DISCUSSION}

The headline result of this work is the refinement of system parameters for 39 transiting hot Jupiters. These are collected and compared to the literature results in Table 4. The stellar parameters and the most relevant planetary and orbital parameters are aggregated in $\mathrm{Ta}-$ ble 5 and in Table 6 , respectively.

We show a comparison between system parameters from this work and from previous studies in Figure 6. Our results are based on a larger number of transits than previous measurements, so we are confident that our results with increased precision (as shown in Figure 7) are preferred over past literature values.

\section{Stellar Physical Parameters:}

Our stellar parameters are in reasonable agreement $(2 \sigma)$ with published results (See Panels a, b, c, d, and e in Figure 6), with the exception of a $2.6 \sigma$ larger $\log g$ value derived for HAT-P-22. The derived system param- 


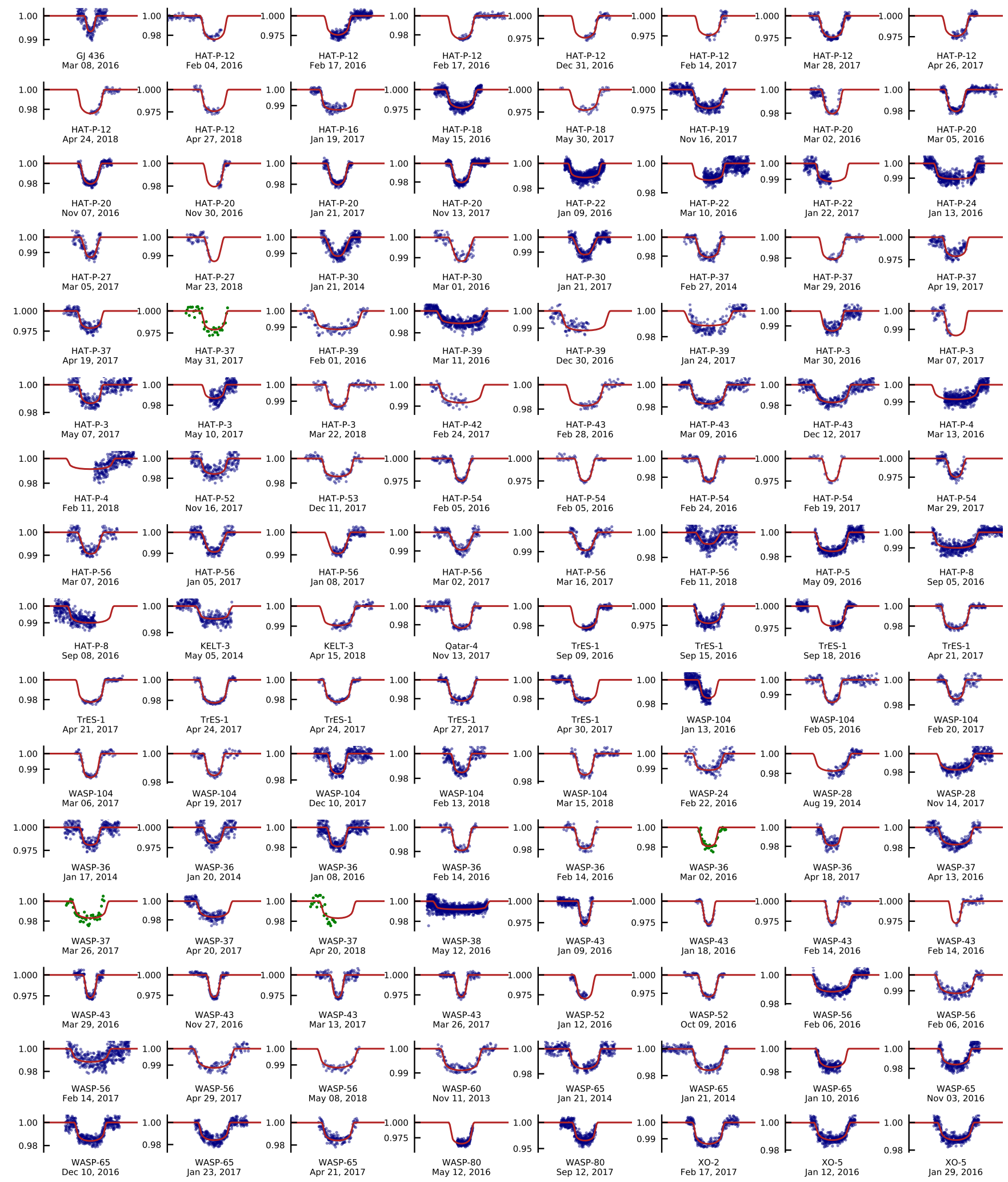

Figure 3. 127 follow-up light curves that we collected for this study. These light curves were fitted simultaneously with the archived photometric, Doppler velocimetric, and spectroscopic data to estimate the system parameters. Four bad light curves are plotted in green. The solid red lines show the best-fitting models. 

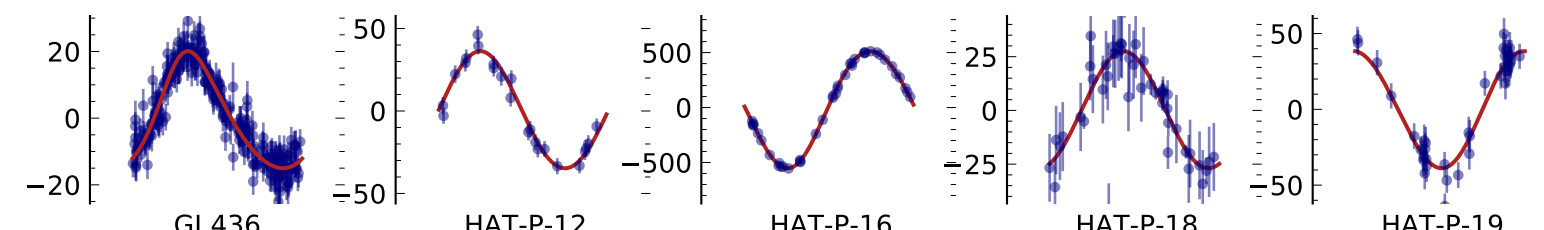

HAT-P-12

HAT-P-16

HAT-P-18

HAT-P-19

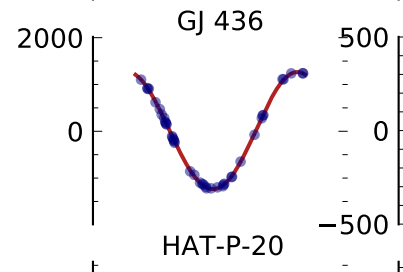

(100)
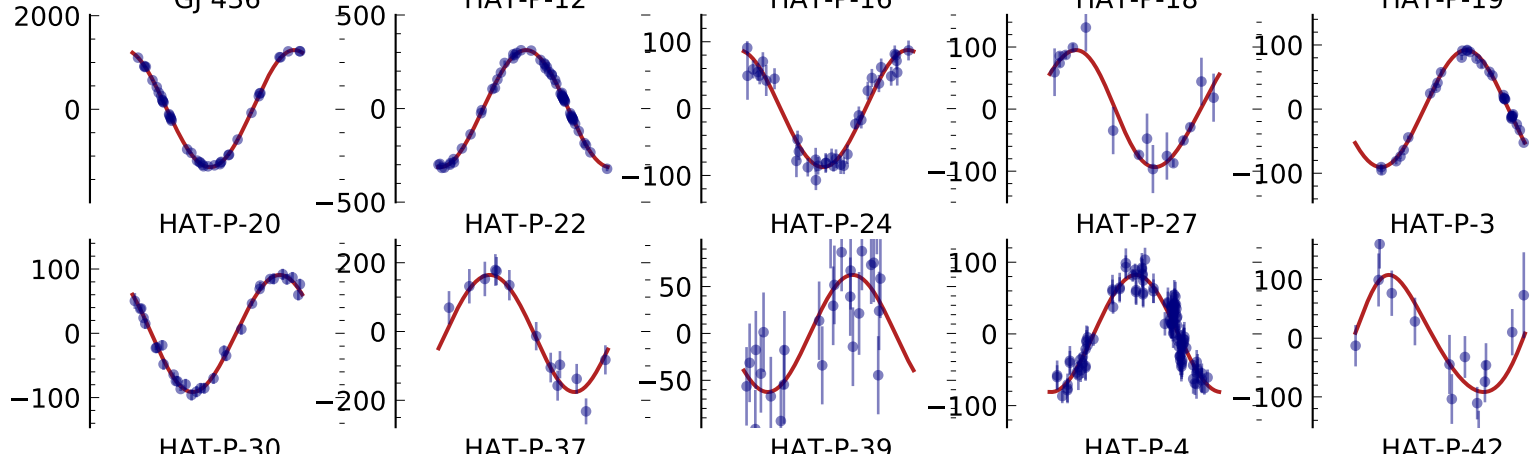

HAT-P-24

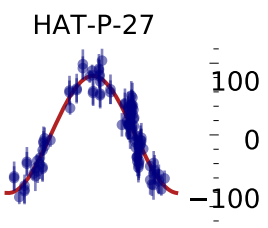

HAT-P-3

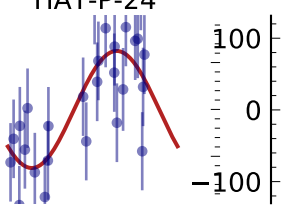

HAT-P-37 200

HAT-P-39

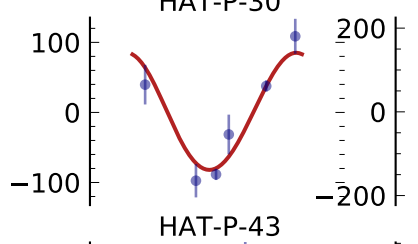

HAT-P-5

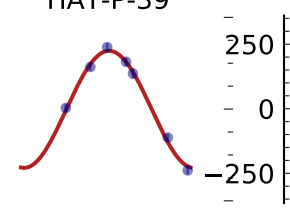

HAT-P-4 $\begin{array}{r}200 \\ 0 \\ -200\end{array} \mid$

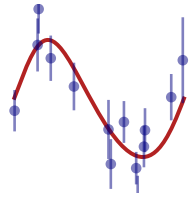

HAT-P-42
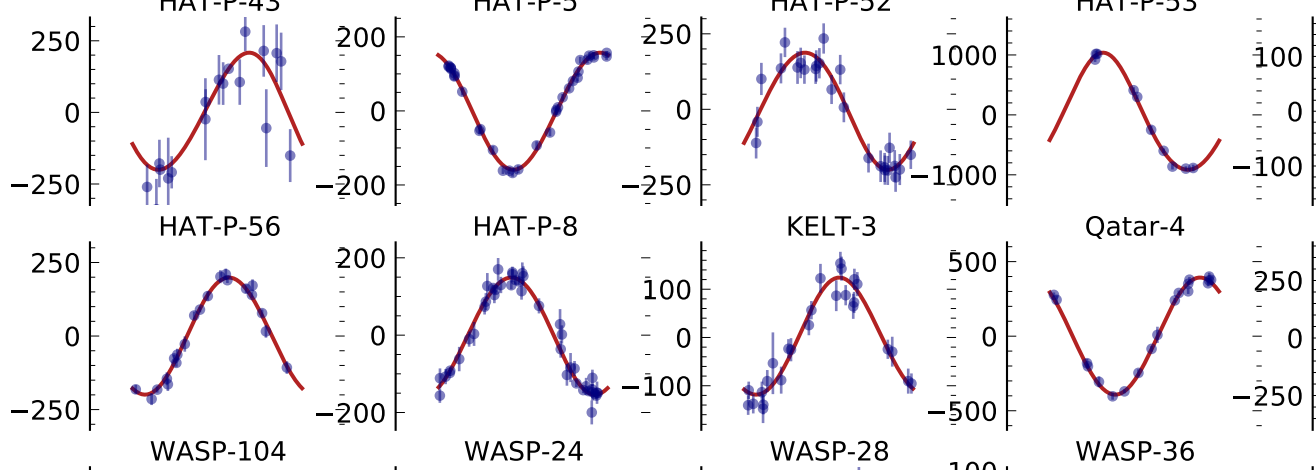

Qatar-4
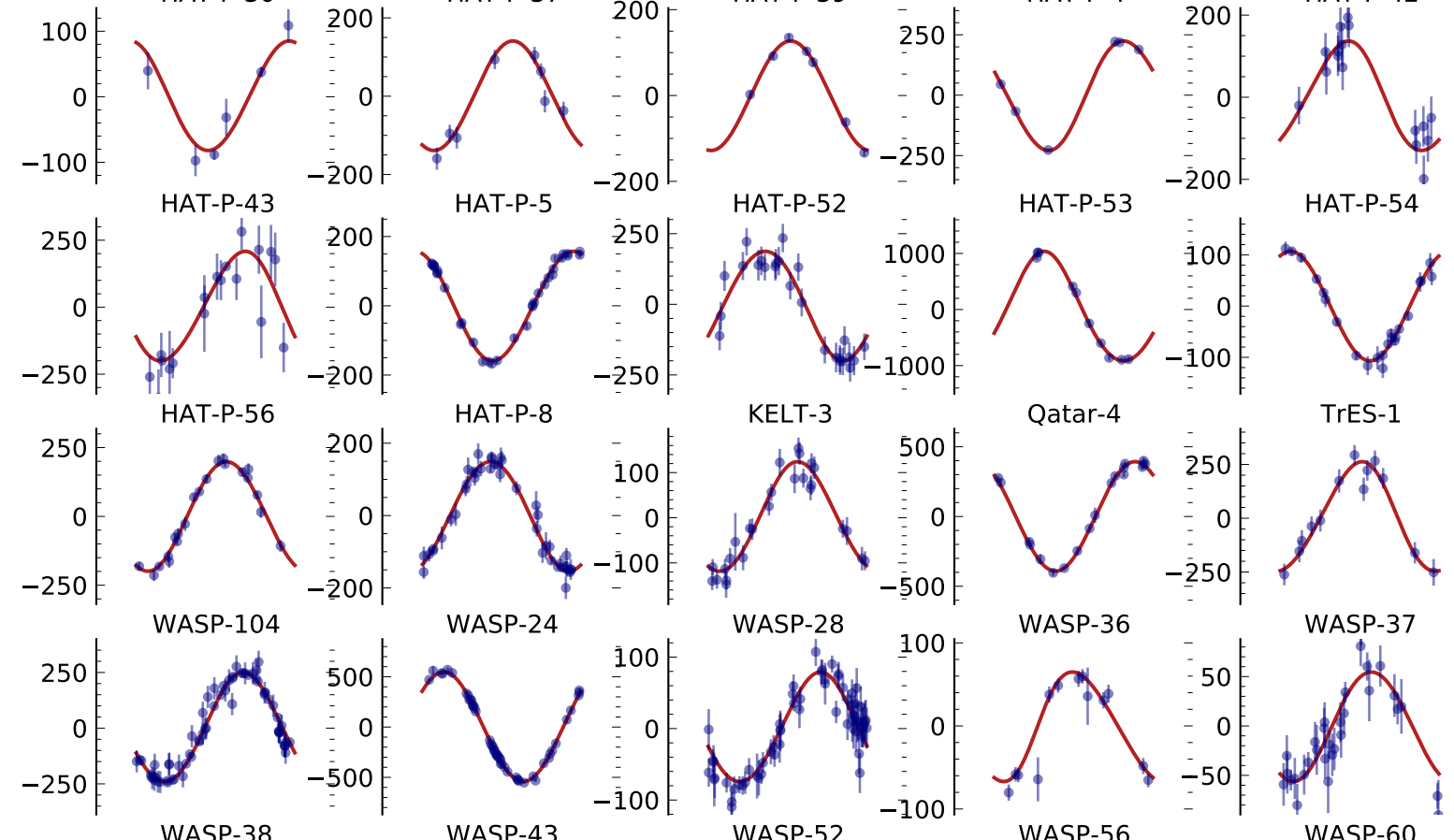

WASP-36

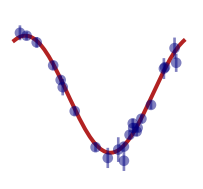

TrES-1
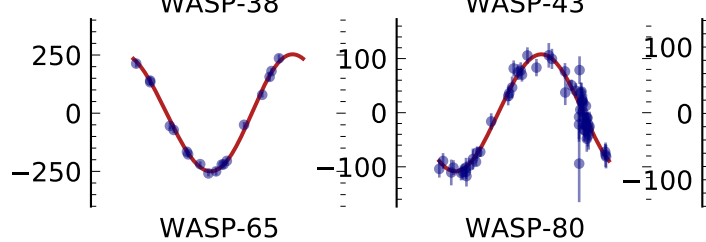

WASP-80
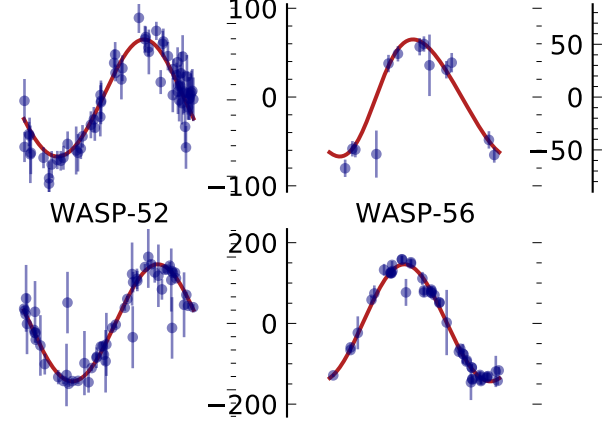

WASP-56
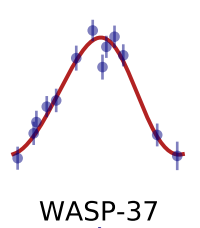

$\mathrm{XO}-2$

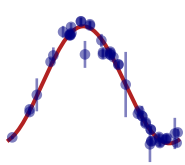

$\mathrm{XO}-5$

Figure 4. Archived Doppler velocimetric measurements $(\mathrm{m} / \mathrm{s})$ of our TEMP targets phase-folded with the updated orbital periods determined in this study. The best-fitting Keplerian orbital solutions from the joint radial-velocity, light-curve, and spectroscopic modeling are overplotted as solid red lines. 

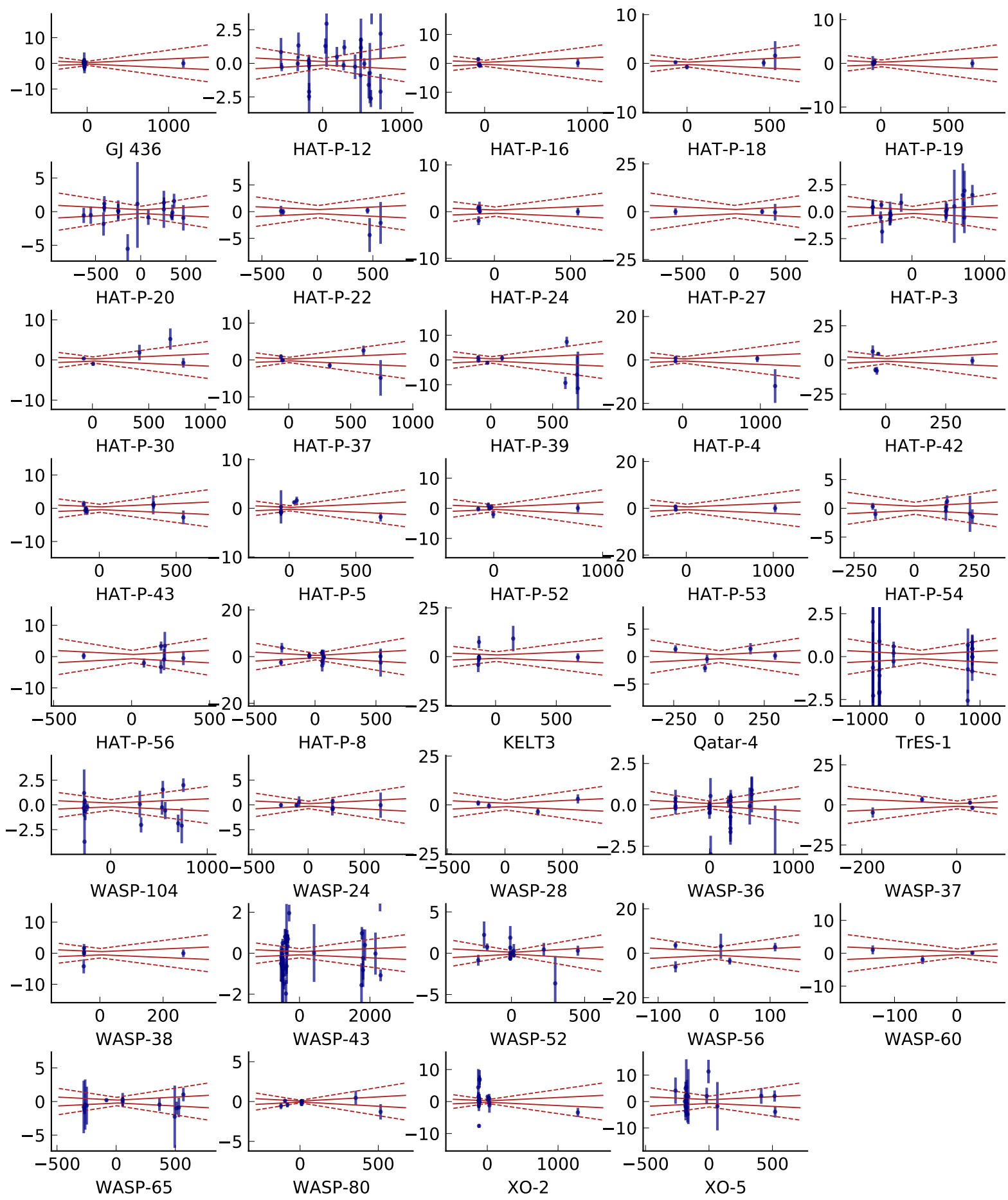

Figure 5. Transit timing variations (mins) for our targets, as compared to the best-fitting ephemerides reported in this study, shown as a function of epoch. The solid (dashed) lines indicate the propagation of $\pm 1 \sigma( \pm 3 \sigma)$ errors in the updated linear ephemerides. No statistically significant TTVs are detected in our sample at levels of $\pm 3 \sigma$. 

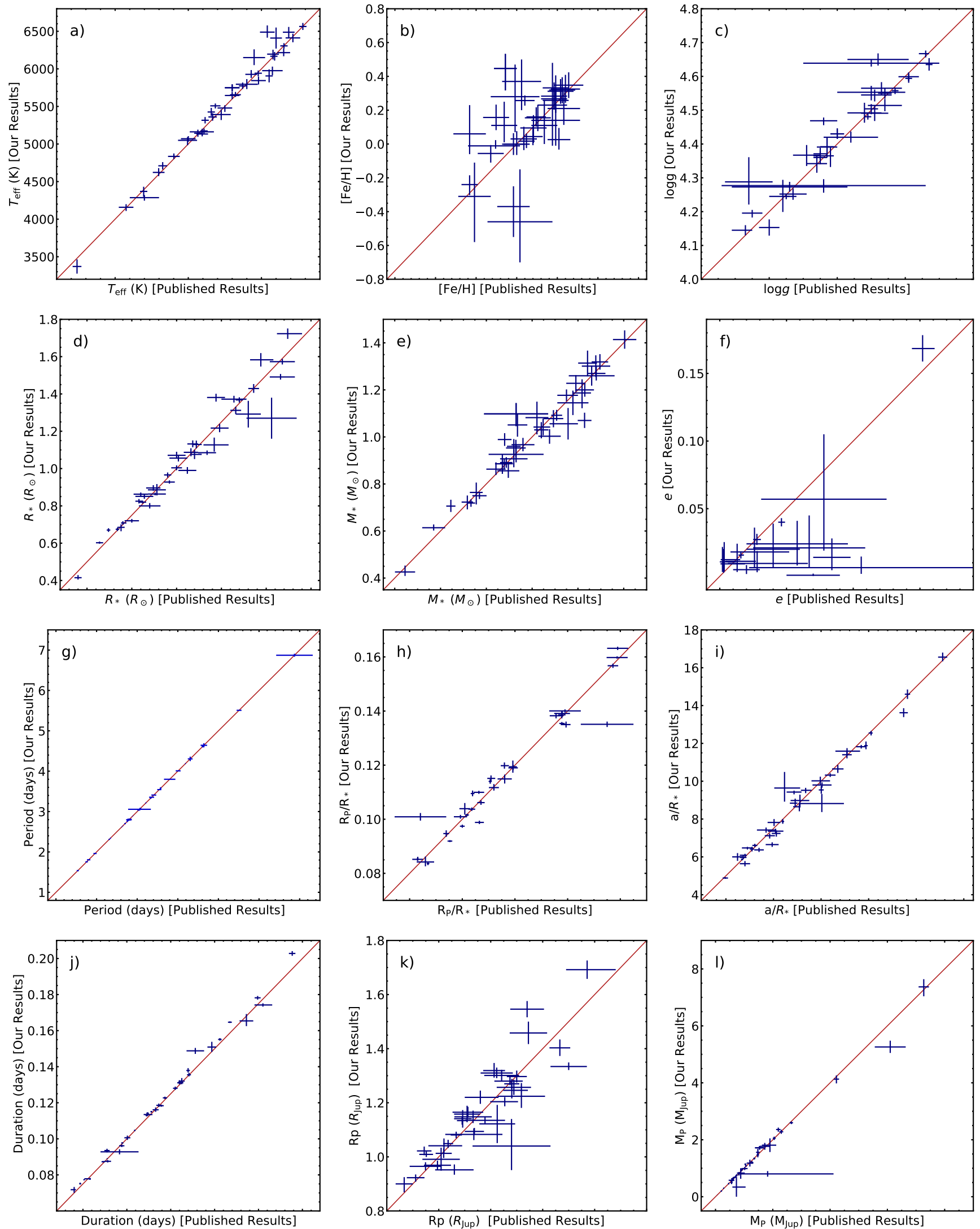

Figure 6. Comparison of the effective temperature ( $T_{\text {eff }}$, Panel a), metallicity $([\mathrm{Fe} / \mathrm{H}]$, Panel b), surface gravity (log $g$, Panel $\mathrm{c})$, stellar radius $\left(R_{*}\right.$, Panel d), stellar mass $\left(M_{*}\right.$, Panel e), orbital eccentricity (e, Panel f), orbital period $(P$, Panel g), planet-to-star radius ratio $\left(R_{\mathrm{P}} / R_{*}\right.$, Panel $\left.\mathrm{h}\right)$, scaled semi-major axis $\left(a / R_{*}\right.$, Panel $\left.\mathrm{i}\right)$, transit duration $\left(T_{14}\right.$, Panel $\left.\mathrm{j}\right)$, planetary radius $\left(R_{\mathrm{P}}\right.$, Panel k), and planetary mass $\left(M_{\mathrm{P}}\right.$, Panel l) derived in this work and in the literature for our 39 target hot Jupiter systems. The solid lines represent exact agreement. 

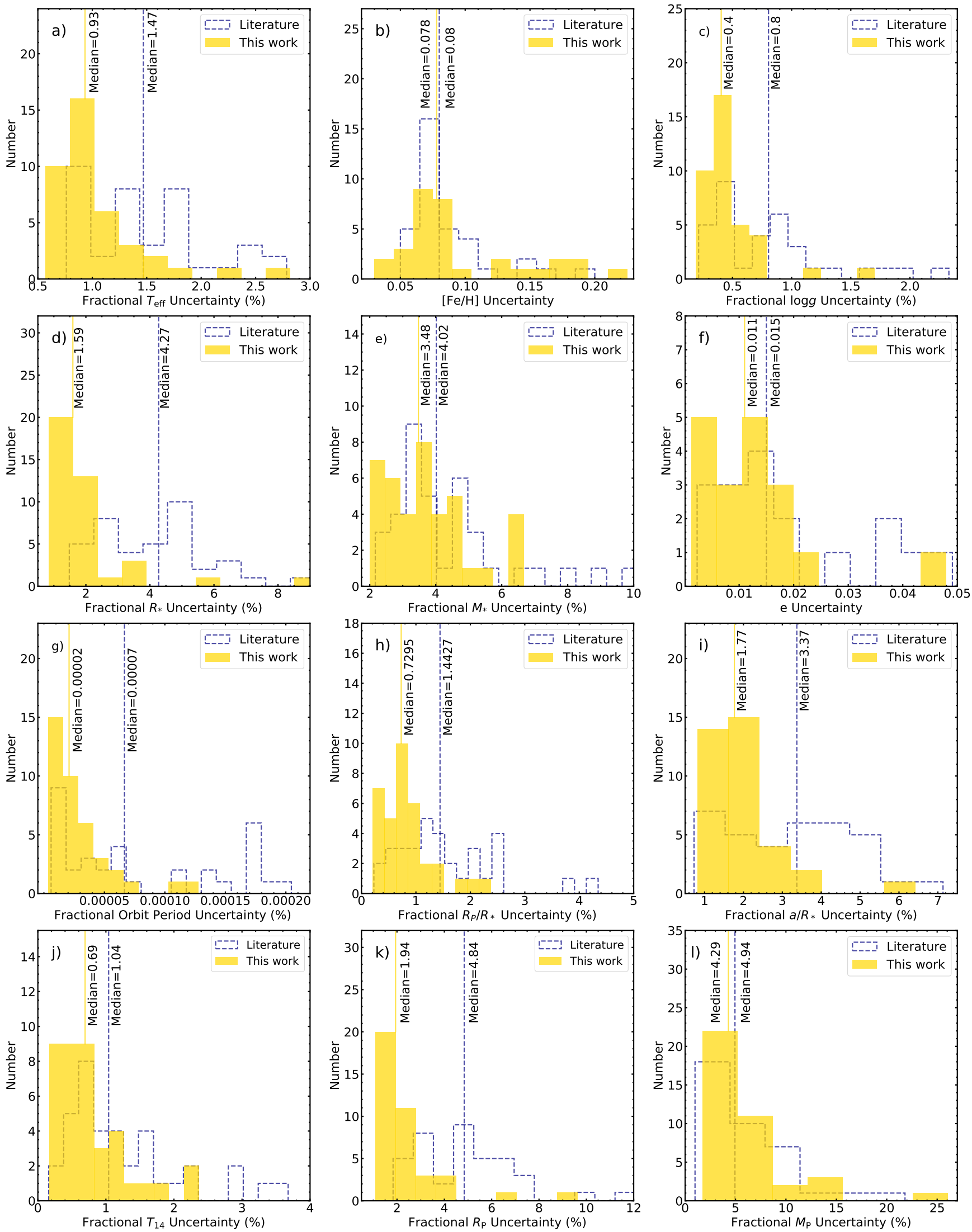

Figure 7. Distribution of uncertainties in fractional effective temperature $\left(\sigma_{T_{\text {eff }}} / T_{\text {eff }}\right.$, Panel a), stellar metallicity $\left(\sigma_{[\mathrm{Fe} / \mathrm{H}]}\right.$, Panels b), fractional surface gravity $\left(\sigma_{\log g} / \log g\right.$, Panel c), fractional stellar radius $\left(\sigma_{R_{*}} / R_{*}\right.$, Panel d), fractional stellar mass $\left(\sigma_{M_{*}} / M_{*}\right.$, Panel e), eccentricity $\left(\sigma_{e}\right.$, Panel f), fractional orbital period $\left(\sigma_{P} / P\right.$, Panel g), fractional planet-to-star radius ratio $\left(\sigma_{R_{\mathrm{P}} / R_{*}} / R_{\mathrm{P}} / R_{*}\right.$, Panel $\left.\mathrm{h}\right)$, fractional scaled semi-major axis $\left(\sigma_{a / R_{*}} / a / R_{*}\right.$, Panel i), fractional transit duration $\left(\sigma_{T_{14}} / T_{14}\right.$, Panel g), fractional planetary radius $\left(\sigma_{R_{\mathrm{P}}} / R_{\mathrm{P}}\right.$, Panel $\left.\mathrm{k}\right)$, and fractional planetary mass $\left(\sigma_{M_{\mathrm{P}}} / M_{\mathrm{P}}\right.$, Panel l) from this work (yellow) compared to the literature values (blue dashed line). 


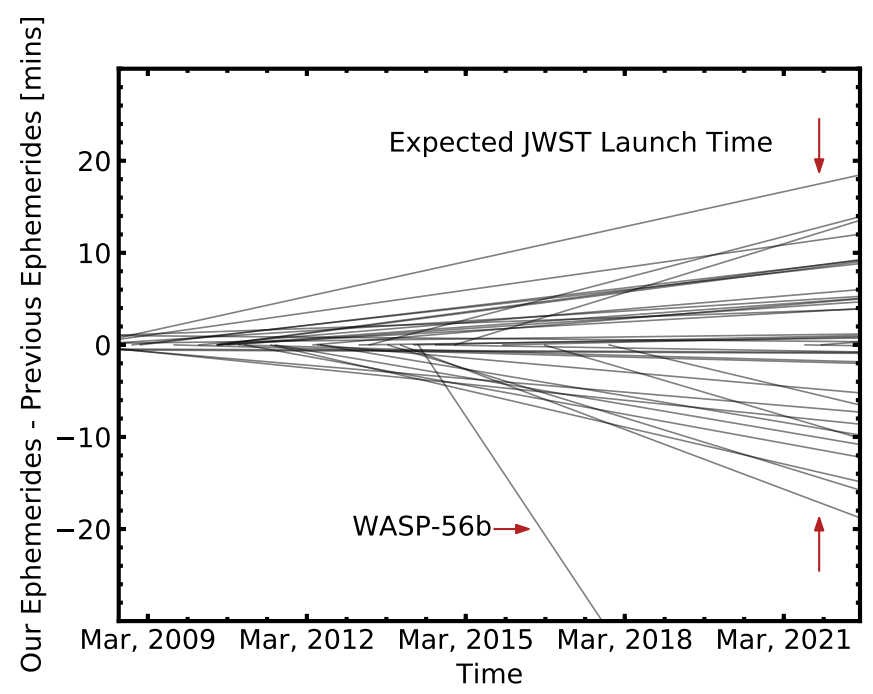

Figure 8. The difference between calculated (predicted) transit windows determined using our updated ephemerides and literature ephemerides. The difference for 29 out of 39 targets are within $10 \mathrm{~min}$ at the time of launch of JWST. For the other 10 targets, our predicted transit mid-times differ by more than $10 \mathrm{~min}$ from the literature ephemerides; for WASP-56b, the deviation amounts to about $72 \mathrm{~min}$.

eters showing $>2 \sigma$ discrepancies are marked in bold in Table 4.

Although $T_{\text {eff }}$ and $[\mathrm{Fe} / \mathrm{H}]$ can be independently constrained from the SED and transit limb darkening, the improvement in precision of these two parameters from our analysis is not significant (See Panels a and b in Figure 7). These parameters are typically best constrained by spectroscopic priors, while our work focuses on extending and refining the photometric baseline for the observed systems.

Compared to literature solutions, we obtained more precise $\log g$ values (See Panel c in Figure 7), since the transit alone provides an additional constraint on $\log g$ through limb darkening. We also significantly improved the precision of the host stellar radii, since a combination of the SED fits to the broad-band photometry, the upper limit on the $V$-band extinction from galactic dust maps, and a parallax constraint from Gaia DR2 precisely determines the bolometric luminosity and therefore stellar radius. All of our stellar radii are constrained to accuracies of better than 3\% (See Panel d in Figure 7). By comparison, previous studies attained the same level of accuracy for only 28 of the 39 systems.

The transit alone provides an independent and additional constraint on the stellar density through its duration (Seager \& Mallén-Ornelas 2003). The stellar mass is, therefore, more precisely constrained in our study (See Panel e in Figure 7) due to our improved stellar radius and stellar density measurements, together with the SED fits to the broadband photometry and MIST fits to $T_{\text {eff }},[\mathrm{Fe} / \mathrm{H}]$, and $\log g$.

\section{Radial Velocity Parameters:}

Since we did not obtain any new radial velocity measurements in this work, it is not surprising that the radial velocity parameters derived from our analysis typically agree with previous results in both errors and values.

Although the eccentricities that we found agree with published values within $2 \sigma$, literature studies are systemically biased towards larger eccentricities (See Panel f in Figure 6) for these nearly circular hot Jupiter systems due to the eccentricity boundary at zero (Wittenmyer et al. 2013, 2019). In this work, instead of sampling directly in $e$, we sample uniformly in $e \cos \omega_{*}$ and $e \sin \omega_{*}$, which helps us to reduce the Lucy-Sweeney bias (Lucy \& Sweeney 1971).

\section{Transit Parameters:}

The majority of the final photometric parameters for our systems - the orbital period $(P$, Panel $g$ in Figure 6 and Figure 7$)$, the planet-to-star radius ratio $\left(R_{\mathrm{P}} / R_{*}\right.$, Panel h in Figure 6 and Figure 7$)$, the scaled semi-major axis $\left(a / R_{*}\right.$, Panel $\mathrm{i}$ in Figure 6 and Figure 7$)$, and the transit duration $\left(T_{14}\right.$, Panel $\mathrm{j}$ in Figure 6 and Figure 7 ) - are in agreement with but more precise than the published values.

For several systems that show $>2 \sigma$ discrepancies in the transit parameters between our analysis and previous literature values (marked in bold in Table 4), we are confident that our results are more reliable since our results are based on more extensive transit data than previous studies.

\section{Planetary Physical Parameters:}

Although we found a larger planetary radius for GJ 436 and a smaller planetary radius for HAT-P-54 compared to previous work, the planetary physical parameters $\left(R_{\mathrm{P}}\right.$ and $\left.M_{\mathrm{P}}\right)$ obtained in this study are generally in reasonable agreement with those from the previous literature (See Panel k and 1 in Figure 6), but with higher precision (see Panel $\mathrm{k}$ and $\mathrm{l}$ in Figure 7). This is especially true for planetary radius, as our results are based on more extensive photometric data. The planetary radii for almost all of our targets (37 out of 39) are determined to accuracies of better than 5\%. By comparison, previous studies attained the same level of accuracy for only 21 of the 39 systems.

\section{Transit Timing Variations:}

Our new photometric observations allow for significant improvements in the orbital ephemerides $\left(T_{0}\right.$ and $P$ ) of each system, which are useful to accurately predict future transit events. By 31 Oct, 2021, the currently 
scheduled James Webb Space Telescope (JWST) (Gardner et al. 2006) launch date, if the literature ephemerides were left unimproved, the accumulated errors of predicted transit mid-times would exceed 1 hour for systems like WASP-56 (see Figure 8), although 29 of our 39 targets are within $10 \mathrm{~min}$ of previous predictions.

The refined orbital ephemerides also enable a search for TTVs. The measured transit mid-times of our targets are consistent with our updated linear ephemerides within $3 \sigma$, except for several outliers (See Figure 5). Although the origin of these outliers could be astrophysical, it is most likely that 1 ). The uncertainties of transit mid-times for the light curves taken under poor weather conditions are underestimated because of the presence of correlated noise or 2). The transit mid-times of very incomplete transits are imprecisely determined. Additional photometry is needed to uncover the nature of these discrepancies.

Our null results agree with previous TTV searches for hot Jupiter companion planets, conducted using both the Kepler dataset (Steffen et al. 2012) and groundbased photometric follow-up programs (for example: TLC, Holman et al. 2006; YETI, Neuhäuser et al. 2011; Trappsit, Gillon et al. 2012; Taste, Nascimbeni et al. 2011; TraMoS, Cortés-Zuleta et al. 2020; TEMP, Wang et al. 2018b; and Mallonn et al. 2019).

\section{Constraints on Additional Planets:}

The absence of statistically significant TTVs provides constraints on the upper mass limits for any putative companion planets in the observed hot Jupiter systems.

Using dynamical simulations with REBOUND (Rein \& Liu 2012; Rein \& Spiegel 2015), we searched for the masses of putative companion planets that would produce TTVs whose RMS values are the same as those measured in Section 3.2. This delineates the massperiod parameter space where additional close-in companion planets would have been detected if they existed, and, conversely, where these planets would have been remained undetected.

Our simulations assume that the putative companion planets are coplanar with the hot Jupiters, and both planets are on circular orbits. Compared with noncoplanar or eccentric orbits, the coplanar and circular configuration induces smaller TTVs and therefore provides a more conservative estimate of the putative companion planets' upper mass limits, as discussed by Agol \& Deck (2016).

We stepped through the period ratio of putative companion planets and known hot Jupiters from 1:5 to 5:1 in 100 steps. The sample resolution was tripled when in proximity to resonances where the largest planetary TTVs are likely to arise (Agol et al. 2005; Hol- man \& Murray 2005). For each step, the approximated upper mass limit of the putative planet was obtained iteratively by linear interpolation with an initial mass guess of $1 \mathrm{M}_{\oplus}$ and with a convergence tolerance of $\left|O_{\text {TTV RMS }}-C_{\text {TTV RMS }}\right|<1$ s.

We did not explore the TTV behavior induced by unstable putative companion planets. The regions of orbital instability were identified using the Mean Exponential Growth factor of Nearby Orbits (MEGNO $\langle Y\rangle$; Cincotta \& Simó 1999, 2000; Cincotta et al. 2003; Hinse et al. 2010), which can efficiently distinguish quasi-periodic $(\langle Y\rangle \rightarrow 2$ as $\mathrm{t} \rightarrow \infty)$ or chaotic motion $(\langle Y\rangle>2$ as $\mathrm{t} \rightarrow$ $\infty)$. The MEGNO maps (see Figure 9 ) are gridded in period ratio-mass space, with 500 evenly spaced values on each axis spanning $0.1<P_{\text {putative planet }} / P_{\text {hot Jupiter }}<$ 3.5 and $10^{-2} \mathrm{M}_{\oplus}<M_{\text {putative planet }}<10^{4} \mathrm{M}_{\oplus}$ for the period ratio of putative companion planet and known hot Jupiter (x-axis) and mass of putative companion planet (y-axis), respectively. At each grid point, the putative companion planet was integrated together with the known hot Jupiter for 1,000 years, at which point we calculated the MEGNO factor $\langle Y\rangle$.

MEGNO also identifies the locations of mean motion resonances. As illustrated in Figure 9, the mass constraints from TTVs are more restrictive at mean-motion resonances (especially the low-order mean-motion resonances) by comparison with general orbital configurations. We rule out the presence of putative companion planets with masses greater than $0.39-5.00 \mathrm{M}_{\oplus}$, $1.23-14.36 \mathrm{M}_{\oplus}, 1.65-21.18 \mathrm{M}_{\oplus}$, and $0.69-6.75 \mathrm{M}_{\oplus}$ near the $1: 2,2: 3,3: 2$, and $2: 1$ resonances, respectively. For a given location in a given hot Jupiter system, the upper mass limit of the putative companion planet $\left(m_{\text {putative planet }}\right)$ is a function of the orbital period of the hot Jupiter $(P)$, the TTV amplitude $(\Delta)$, and the host stellar mass $\left(M_{*}\right)$, roughly scaling as $m_{\text {putative planet }}=$ $P \cdot \Delta / M_{*}$. Since the systems in our sample have a similar range of stellar mass ( $M_{*}$ ranging from 0.426 to $1.41 \mathrm{M}_{\odot}$ ) and orbital period $(P$ ranging from 0.81 to 6.87 days), the upper limits of the putative companion planet masses are dominantly set by the TTV amplitudes ( $\Delta$ ranging from 11 to $585 \mathrm{~s}$ ) of our hot Jupiters.

The absence of nearby resonant companion planets in hot Jupiter systems is inconsistent with the conventional expectation from disk migration. Although disk migration does not provide a precise quantitative prediction of the typical occurrence rate of resonant pairs, the absence of resonant companion planets across the hot Jupiter sample, as suggested by this work and previous publications (Steffen et al. 2012; Montalto et al. 2012), strongly disfavors disk migration. This absence is compatible with violent high-eccentricity tidal migra- 
tion, which ejects any original close-in companion planets. We cannot rule out in-situ formation, since, although in-situ formation tends to form hot Jupiters accompanied by nearby planets (Boley et al. 2016; Batygin et al. 2016), those planets are not necessarily in or near mean motion resonance.

A dearth of additional transit signal detections in the Kepler hot Jupiter sample initially appeared to place a very strong constraint on the occurrence rate of nonresonant companion planets in those systems (Steffen et al. 2012; Huang et al. 2017). However, these findings, which are generally quoted as evidence for the higheccentricity origin of hot Jupiters, could instead result from observational bias, since the companion planets can hide in exterior and/or inclined orbits (Millholland et al. 2016), or in the observational noise. Previously hidden close-in companion planets in existing hot Jupiter systems have begun to be discovered via the transit method as better photometric precision is achieved (WASP-47, Becker et al. 2015; Kepler-730, Cañas et al. 2019; TOI1130, Huang et al. 2020), as well as through the radial velocity method (WASP-148: Hébrard et al. 2020). These systems cannot be explained by high-eccentricity tidal migration, but they are consistent with in-situ formation. 


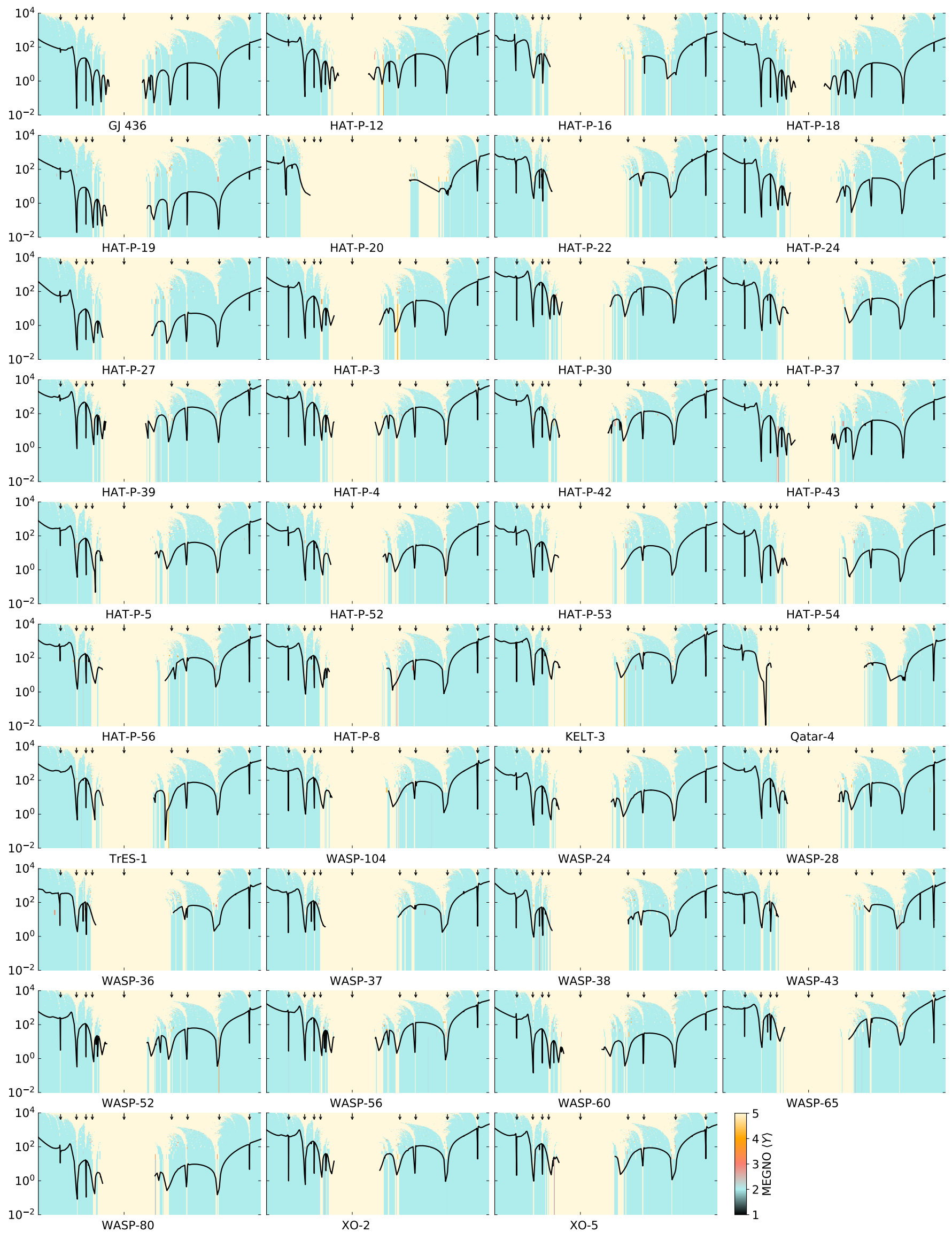

Figure 9. Upper mass limits $\left(\mathrm{M}_{\oplus}\right)$ as a function of the orbital period ratio of the putative companion planets and the transiting hot Jupiters. The black solid curves represent the mass-period region where putative companion planets would produce TTVs with RMS values matching those that we measured for our targets. The mass constraints are most restrictive near the low-order mean-motion resonances (marked by arrows). We use MEGNO Factor $(\langle Y\rangle)$ to identify quasi-periodic regions $(\langle Y\rangle=2$, color coded blue), as well as chaotic and possibly unstable regions $(\langle Y\rangle \neq 2$, color coded black, red, and yellow). 
Table 1. Log of Observations of the TEMP Targets Presented in This Work

\begin{tabular}{|c|c|c|c|c|c|c|c|}
\hline Planet & $\begin{array}{l}\text { Date } \\
\text { (UTC) }\end{array}$ & $\begin{array}{l}\text { Time } \\
\text { (UTC) }\end{array}$ & Telescope & $\begin{array}{c}\text { Typical Exposure Time } \\
(\mathrm{s})\end{array}$ & $\mathrm{N}_{\text {data }}$ & PNR & Quality \\
\hline GJ $436 \mathrm{~b}$ & Mar 08, 2016 & $13: 17: 15-15: 50: 10$ & Xinglong $60 / 90 \mathrm{~cm}$ Schmidt & 40 & 174 & 0.12 & Golden \\
\hline \multirow[t]{9}{*}{ HAT-P-12 b } & Feb 04, 2016 & $17: 46: 07-22: 19: 55$ & Xinglong $60 / 90 \mathrm{~cm}$ Schmidt & 200 & 76 & 0.32 & Mediocre \\
\hline & Feb 17, 2016 & $17: 37: 22-21: 05: 46$ & Xinglong $60 \mathrm{~cm}$ & 30 & 323 & 0.15 & Golden \\
\hline & Feb 17, 2016 & $17: 57: 53-21: 53: 21$ & Xinglong $60 / 90 \mathrm{~cm}$ Schmidt & 140 & 94 & 0.17 & Golden \\
\hline & Dec 31,2016 & $20: 25: 13-22: 31: 45$ & Xinglong $60 / 90 \mathrm{~cm}$ Schmidt & 150 & 48 & 0.21 & Mediocre \\
\hline & Feb 14, 2017 & $19: 49: 37-21: 33: 24$ & Xinglong $60 / 90 \mathrm{~cm}$ Schmidt & 80 & 41 & 0.29 & Mediocre \\
\hline & Mar 28, 2017 & $12: 31: 25-15: 59: 05$ & Xinglong $60 \mathrm{~cm}$ & 60 & 134 & 0.20 & Mediocre \\
\hline & Apr 26,2017 & $12: 11: 48-14: 18: 28$ & Xinglong 60/90 cm Schmidt & 80 & 73 & 0.27 & Mediocre \\
\hline & Apr 24, 2018 & $14: 15: 57-16: 48: 05$ & Xinglong $60 \mathrm{~cm}$ & 180 & 44 & 0.21 & Mediocre \\
\hline & Apr 27, 2018 & $17: 40: 03-19: 48: 32$ & Xinglong $60 \mathrm{~cm}$ & 180 & 36 & 0.25 & Mediocre \\
\hline HAT-P-16 b & $\operatorname{Jan} 19,2017$ & $10: 16: 44-13: 17: 18$ & Xinglong 60/90 cm Schmidt & 80 & 106 & 0.13 & Golden \\
\hline \multirow[t]{2}{*}{ HAT-P-18 b } & May 15,2016 & $12: 30: 12-16: 33: 04$ & Xinglong $60 \mathrm{~cm}$ & 30 & 397 & 0.17 & Golden \\
\hline & May 30, 2017 & $14: 01: 18-18: 10: 23$ & Xinglong $60 \mathrm{~cm}$ & 30 & 34 & 0.41 & Mediocre \\
\hline HAT-P-19 b & Nov 16, 2017 & $10: 25: 29-15: 18: 45$ & Xinglong $60 \mathrm{~cm}$ & 25 & 363 & 0.22 & Mediocre \\
\hline \multirow[t]{6}{*}{ HAT-P-20 b } & Mar 02, 2016 & $14: 41: 46-17: 19: 44$ & Xinglong $60 / 90 \mathrm{~cm}$ Schmidt & 60 & 90 & 0.26 & Mediocre \\
\hline & Mar 05, 2016 & $12: 16: 08-17: 04: 46$ & Xinglong $60 / 90 \mathrm{~cm}$ Schmidt & 55 & 243 & 0.13 & Golden \\
\hline & Nov 07,2016 & 19:09:22-22:00:40 & Xinglong $60 \mathrm{~cm}$ & 30 & 195 & 0.11 & Golden \\
\hline & Nov 30,2016 & $20: 33: 31-21: 37: 17$ & Xinglong $60 / 90 \mathrm{~cm}$ Schmidt & 90 & 35 & 0.16 & Golden \\
\hline & Jan 21,2017 & $13: 09: 29-15: 38: 39$ & Xinglong $60 \mathrm{~cm}$ & 30 & 165 & 0.13 & Golden \\
\hline & Nov 13, 2017 & $16: 52: 34-20: 33: 30$ & Xinglong $60 \mathrm{~cm}$ & 18 & 321 & 0.13 & Golden \\
\hline \multirow[t]{3}{*}{ HAT-P-22 b } & Jan 09, 2016 & $15: 27: 06-18: 51: 35$ & Xinglong $60 \mathrm{~cm}$ & 10 & 770 & 0.09 & Golden \\
\hline & Mar 10, 2016 & $17: 32: 40-21: 23: 25$ & Xinglong $60 \mathrm{~cm}$ & 20 & 539 & 0.13 & Golden \\
\hline & $\operatorname{Jan} 22,2017$ & $15: 49: 49-18: 07: 29$ & Xinglong $60 \mathrm{~cm}$ & 20 & 181 & 0.14 & Golden \\
\hline HAT-P-24 b & Jan 13,2016 & $15: 04: 46-20: 11: 18$ & Xinglong $60 \mathrm{~cm}$ & 20 & 570 & 0.11 & Golden \\
\hline \multirow[t]{2}{*}{ HAT-P-27 b } & Mar 05, 2017 & $17: 32: 14-19: 57: 17$ & Xinglong $60 / 90 \mathrm{~cm}$ Schmidt & 70 & 94 & 0.21 & Mediocre \\
\hline & Mar 23, 2018 & $16: 28: 44-18: 28: 21$ & Xinglong $60 \mathrm{~cm}$ & 130 & 47 & 0.23 & Mediocre \\
\hline \multirow[t]{5}{*}{ HAT-P-3 b } & Mar 30, 2016 & $12: 50: 45-16: 02: 01$ & Xinglong $60 / 90 \mathrm{~cm}$ Schmidt & 60 & 180 & 0.19 & Golden \\
\hline & Mar 07, 2017 & $15: 35: 15-17: 11: 25$ & Xinglong 60/90 cm Schmidt & 110 & 48 & 0.21 & Mediocre \\
\hline & May 07,2017 & $13: 13: 30-18: 36: 43$ & Xinglong $60 \mathrm{~cm}$ & 40 & 310 & 0.21 & Mediocre \\
\hline & May 10, 2017 & $12: 18: 24-14: 34: 42$ & Xinglong $60 \mathrm{~cm}$ & 10 & 221 & 0.21 & Mediocre \\
\hline & Mar 22, 2018 & $12: 19: 33-17: 37: 22$ & Xinglong $60 \mathrm{~cm}$ & 170 & 91 & 0.15 & Golden \\
\hline \multirow[t]{3}{*}{ HAT-P-30 b } & Jan 21,2014 & $13: 52: 02-15: 38: 27$ & Xinglong $60 \mathrm{~cm}$ & 30 & 299 & 0.13 & Golden \\
\hline & Mar 01, 2016 & $14: 06: 32-17: 28: 20$ & Xinglong 60/90 cm Schmidt & 100 & 80 & 0.20 & Golden \\
\hline & $\operatorname{Jan} 21,2017$ & $15: 43: 00-19: 14: 28$ & Xinglong $60 \mathrm{~cm}$ & 18 & 301 & 0.12 & Golden \\
\hline \multirow[t]{4}{*}{ HAT-P-37 b } & Feb 27, 2014 & $18: 35: 54-22: 01: 09$ & Xinglong $60 \mathrm{~cm}$ & 90 & 120 & 0.25 & Mediocre \\
\hline & Mar 29, 2016 & $17: 59: 16-21: 01: 16$ & Xinglong $60 / 90 \mathrm{~cm}$ Schmidt & 180 & 56 & 0.21 & Mediocre \\
\hline & Apr 19, 2017 & $17: 11: 22-20: 22: 21$ & Xinglong $60 \mathrm{~cm}$ & 50 & 146 & 0.27 & Mediocre \\
\hline & May 31, 2017 & $16: 09: 27-19: 41: 51$ & Xinglong $60 \mathrm{~cm}$ & 40 & 39 & 0.58 & Bad \\
\hline
\end{tabular}

Table 1 continued 
Table 1 (continued)

\begin{tabular}{|c|c|c|c|c|c|c|c|}
\hline Planet & $\begin{array}{c}\text { Date } \\
\text { (UTC) }\end{array}$ & $\begin{array}{c}\text { Time } \\
\text { (UTC) }\end{array}$ & Telescope & $\begin{array}{c}\text { Typical Exposure Time } \\
(\mathrm{s})\end{array}$ & $\mathrm{N}_{\text {data }}$ & PNR & Quality \\
\hline \multirow[t]{4}{*}{ HAT-P-39 b } & Feb 01, 2016 & $11: 54: 55-17: 38: 54$ & Xinglong 60/90 cm Schmidt & 160 & 109 & 0.28 & Mediocre \\
\hline & Mar 11, 2016 & $11: 34: 31-16: 53: 11$ & Xinglong $60 \mathrm{~cm}$ & 30 & 529 & 0.16 & Golden \\
\hline & Dec 30, 2016 & $15: 05: 30-18: 22: 49$ & Xinglong 60/90 cm Schmidt & 120 & 89 & 0.28 & Mediocre \\
\hline & Jan 24,2017 & $11: 49: 28-16: 40: 59$ & Xinglong $60 \mathrm{~cm}$ & 60 & 170 & 0.32 & Mediocre \\
\hline \multirow[t]{2}{*}{ HAT-P-4 b } & Mar 13, 2016 & $17: 42: 52-21: 29: 26$ & Xinglong $60 \mathrm{~cm}$ & 20 & 534 & 0.09 & Golden \\
\hline & Feb 11, 2018 & $17: 39: 19-21: 02: 29$ & Xinglong $60 \mathrm{~cm}$ & 20 & 254 & 0.24 & Mediocre \\
\hline HAT-P-42 b & Feb 24, 2017 & $15: 37: 30-18: 40: 52$ & Xinglong $60 / 90 \mathrm{~cm}$ Schmidt & 200 & 55 & 0.19 & Golden \\
\hline \multirow[t]{3}{*}{ HAT-P-43 b } & Feb 28, 2016 & $13: 40: 38-17: 22: 45$ & Xinglong 60/90 cm Schmidt & 200 & 61 & 0.23 & Mediocre \\
\hline & Mar 09, 2016 & $11: 32: 30-17: 33: 03$ & Xinglong $60 \mathrm{~cm}$ & 80 & 233 & 0.19 & Golden \\
\hline & Dec 12, 2017 & $16: 10: 28-22: 34: 57$ & Xinglong $60 \mathrm{~cm}$ & 50 & 307 & 0.24 & Mediocre \\
\hline HAT-P-5 b & May 09,2016 & 16:08:34-20:18:52 & Xinglong $60 \mathrm{~cm}$ & 20 & 435 & 0.13 & Golden \\
\hline HAT-P-52 b & Nov 16, 2017 & $16: 12: 38-20: 18: 02$ & Xinglong $60 \mathrm{~cm}$ & 40 & 222 & 0.34 & Mediocre \\
\hline HAT-P-53 b & Dec 11, 2017 & $10: 22: 06-15: 16: 34$ & Xinglong $60 \mathrm{~cm}$ & 180 & 85 & 0.25 & Mediocre \\
\hline \multirow[t]{5}{*}{ HAT-P-54 b } & Feb 05, 2016 & 10:58:23-14:26:31 & Xinglong $60 \mathrm{~cm}$ & 120 & 93 & 0.19 & Golden \\
\hline & Feb 05, 2016 & $11: 06: 12-15: 13: 04$ & Xinglong $60 / 90 \mathrm{~cm}$ Schmidt & 290 & 48 & 0.27 & Mediocre \\
\hline & Feb 24, 2016 & 11:11:08-14:51:16 & Xinglong $60 / 90 \mathrm{~cm}$ Schmidt & 160 & 78 & 0.23 & Mediocre \\
\hline & Feb 19, 2017 & $11: 12: 32-14: 09: 23$ & Xinglong $60 \mathrm{~cm}$ & 60 & 25 & 0.39 & Mediocre \\
\hline & Mar 29, 2017 & $11: 27: 29-13: 55: 49$ & Xinglong $60 \mathrm{~cm}$ & 50 & 110 & 0.30 & Mediocre \\
\hline \multirow[t]{6}{*}{ HAT-P-56 b } & Mar 07, 2016 & $11: 18: 29-15: 34: 21$ & Xinglong 60/90 cm Schmidt & 60 & 165 & 0.12 & Golden \\
\hline & Jan 05,2017 & $16: 02: 17-19: 51: 19$ & Xinglong $60 / 90 \mathrm{~cm}$ Schmidt & 60 & 191 & 0.11 & Golden \\
\hline & Jan 08,2017 & $12: 37: 08-15: 25: 31$ & Xinglong $60 / 90 \mathrm{~cm}$ Schmidt & 70 & 121 & 0.09 & Golden \\
\hline & Mar 02, 2017 & $11: 22: 09-15: 12: 53$ & Xinglong $60 / 90 \mathrm{~cm}$ Schmidt & 80 & 141 & 0.13 & Golden \\
\hline & Mar 16, 2017 & $11: 16: 49-14: 54: 48$ & Xinglong $60 / 90 \mathrm{~cm}$ Schmidt & 80 & 121 & 0.14 & Golden \\
\hline & Feb 11, 2018 & $13: 17: 10-17: 37: 21$ & Xinglong $60 \mathrm{~cm}$ & 10 & 423 & 0.23 & Mediocre \\
\hline \multirow[t]{2}{*}{ HAT-P-8 b } & Sep 05, 2016 & $11: 43: 00-17: 56: 37$ & Xinglong $60 \mathrm{~cm}$ & 20 & 505 & 0.13 & Golden \\
\hline & Sep 08, 2016 & $12: 28: 56-16: 00: 13$ & Xinglong $60 \mathrm{~cm}$ & 14 & 347 & 0.16 & Golden \\
\hline \multirow[t]{2}{*}{ KELT-3 b } & May 05, 2014 & 12:03:08-16:44:55 & Xinglong $60 \mathrm{~cm}$ & 60 & 265 & 0.23 & Mediocre \\
\hline & Apr 15, 2018 & 12:33:32-16:00:34 & Xinglong $60 \mathrm{~cm}$ & 100 & 92 & 0.09 & Golden \\
\hline Qatar-4 b & Nov 13,2017 & 10:24:09-15:07:17 & Xinglong $60 \mathrm{~cm}$ & 100 & 130 & 0.24 & Mediocre \\
\hline \multirow[t]{9}{*}{ TrES-1 b } & Sep 09, 2016 & 12:01:48-14:30:25 & Xinglong $60 \mathrm{~cm}$ & 60 & 119 & 0.12 & Golden \\
\hline & Sep 15, 2016 & $12: 26: 32-14: 58: 10$ & Xinglong $60 \mathrm{~cm}$ & 18 & 216 & 0.16 & Golden \\
\hline & Sep 18, 2016 & $11: 22: 10-16: 27: 05$ & Xinglong $60 \mathrm{~cm}$ & 22 & 218 & 0.16 & Golden \\
\hline & Apr 21, 2017 & 15:58:58-20:11:03 & Xinglong $60 \mathrm{~cm}$ & 100 & 116 & 0.16 & Golden \\
\hline & Apr 21, 2017 & $16: 55: 05-20: 22: 28$ & Xinglong $60 / 90 \mathrm{~cm}$ Schmidt & 160 & 73 & 0.13 & Golden \\
\hline & Apr 24, 2017 & $16: 39: 39-20: 12: 48$ & Xinglong $60 \mathrm{~cm}$ & 100 & 93 & 0.12 & Golden \\
\hline & Apr 24,2017 & $16: 43: 20-20: 17: 45$ & Xinglong $60 / 90 \mathrm{~cm}$ Schmidt & 160 & 76 & 0.16 & Golden \\
\hline & Apr 27, 2017 & $17: 06: 20-20: 26: 14$ & Xinglong $60 / 90 \mathrm{~cm}$ Schmidt & 80 & 132 & 0.16 & Golden \\
\hline & Apr 30, 2017 & $16: 57: 51-20: 18: 08$ & Xinglong $60 / 90 \mathrm{~cm}$ Schmidt & 70 & 145 & 0.14 & Golden \\
\hline \multirow[t]{2}{*}{ WASP-104 b } & Jan 13,2016 & $20: 14: 32-22: 24: 53$ & Xinglong $60 \mathrm{~cm}$ & 15 & 367 & 0.12 & Golden \\
\hline & Feb 05, 2016 & 15:40:51-21:46:28 & Xinglong $60 / 90 \mathrm{~cm}$ Schmidt & 120 & 144 & 0.17 & Golden \\
\hline
\end{tabular}

Table 1 continued 
Table 1 (continued)

\begin{tabular}{|c|c|c|c|c|c|c|c|}
\hline Planet & $\begin{array}{c}\text { Date } \\
\text { (UTC) }\end{array}$ & $\begin{array}{c}\text { Time } \\
\text { (UTC) }\end{array}$ & Telescope & $\begin{array}{c}\text { Typical Exposure Time } \\
\text { (s) }\end{array}$ & $\mathrm{N}_{\text {data }}$ & PNR & Quality \\
\hline & Feb 20, 2017 & 14:33:04-18:14:48 & Xinglong $60 / 90 \mathrm{~cm}$ Schmidt & 110 & 111 & 0.22 & Mediocre \\
\hline & Mar 06, 2017 & $15: 48: 40-19: 12: 57$ & Xinglong $60 / 90 \mathrm{~cm}$ Schmidt & 120 & 73 & 0.15 & Golden \\
\hline & Apr 19, 2017 & $12: 26: 07-16: 36: 06$ & Xinglong $60 / 90 \mathrm{~cm}$ Schmidt & 160 & 80 & 0.18 & Golden \\
\hline & Dec 10, 2017 & $17: 44: 45-22: 39: 58$ & Xinglong $60 \mathrm{~cm}$ & 30 & 325 & 0.17 & Golden \\
\hline & Feb 13, 2018 & 17:01:06-20:38:11 & Xinglong $60 \mathrm{~cm}$ & 25 & 208 & 0.20 & Golden \\
\hline & Mar 15, 2018 & $13: 33: 15-17: 38: 18$ & Xinglong $60 \mathrm{~cm}$ & 139 & 83 & 0.17 & Golden \\
\hline WASP-24 b & Feb 22, 2016 & $17: 57: 38-22: 08: 20$ & Xinglong $60 / 90 \mathrm{~cm}$ Schmidt & 80 & 124 & 0.26 & Mediocre \\
\hline \multirow[t]{2}{*}{ WASP-28 b } & Aug 19, 2014 & $15: 16: 26-18: 06: 52$ & Xinglong $60 \mathrm{~cm}$ & 90 & 102 & 0.24 & Mediocre \\
\hline & Nov 14,2017 & 11:05:38-15:30:57 & Xinglong $60 \mathrm{~cm}$ & 25 & 313 & 0.23 & Mediocre \\
\hline \multirow[t]{7}{*}{ WASP-36 b } & Jan 17,2014 & $14: 39: 45-19: 20: 42$ & Xinglong $60 \mathrm{~cm}$ & 60 & 264 & 0.29 & Mediocre \\
\hline & Jan 20,2014 & $17: 07: 56-20: 31: 16$ & Xinglong $60 \mathrm{~cm}$ & 60 & 188 & 0.36 & Mediocre \\
\hline & Jan 08,2016 & $15: 45: 33-20: 07: 08$ & Xinglong $60 \mathrm{~cm}$ & 40 & 337 & 0.22 & Mediocre \\
\hline & Feb 14, 2016 & $13: 10: 45-16: 17: 46$ & Xinglong $60 \mathrm{~cm}$ & 150 & 69 & 0.21 & Mediocre \\
\hline & Feb 14, 2016 & 13:11:30-16:05:21 & Xinglong $60 / 90 \mathrm{~cm}$ Schmidt & 160 & 60 & 0.27 & Mediocre \\
\hline & Mar 02, 2016 & 12:01:44-14:19:24 & Xinglong $60 / 90 \mathrm{~cm}$ Schmidt & 250 & 33 & 0.47 & Bad \\
\hline & Apr 18, 2017 & 11:51:53-13:43:47 & Xinglong $60 \mathrm{~cm}$ & 50 & 92 & 0.29 & Mediocre \\
\hline \multirow[t]{4}{*}{ WASP-37 b } & Apr 13, 2016 & 15:50:06-20:26:14 & Xinglong $60 \mathrm{~cm}$ & 55 & 249 & 0.22 & Mediocre \\
\hline & Mar 26, 2017 & $16: 37: 22-19: 50: 35$ & Xinglong $60 \mathrm{~cm}$ & 40 & 43 & 0.72 & Bad \\
\hline & Apr 20,2017 & $17: 13: 06-20: 38: 04$ & Xinglong $60 \mathrm{~cm}$ & 40 & 180 & 0.21 & Mediocre \\
\hline & Apr 20, 2018 & 15:02:26-17:08:44 & Xinglong $60 \mathrm{~cm}$ & 249 & 26 & 0.71 & $\mathrm{Bad}$ \\
\hline WASP-38 b & May 12,2016 & $17: 31: 50-17: 31: 32$ & Xinglong $60 \mathrm{~cm}$ & 12 & 1056 & 0.11 & Golden \\
\hline \multirow[t]{8}{*}{ WASP-43 b } & Jan 09, 2016 & 19:42:49-22:43:56 & Xinglong $60 \mathrm{~cm}$ & 45 & 219 & 0.2 & Golden \\
\hline & Jan 18,2016 & 19:42:52-21:18:44 & Xinglong $60 \mathrm{~cm}$ & 150 & 38 & 0.15 & Golden \\
\hline & Feb 14, 2016 & $16: 24: 49-18: 37: 08$ & Xinglong $60 \mathrm{~cm}$ & 150 & 51 & 0.27 & Mediocre \\
\hline & Feb 14, 2016 & $17: 07: 08-19: 24: 23$ & Xinglong $60 / 90 \mathrm{~cm}$ Schmidt & 160 & 49 & 0.30 & Mediocre \\
\hline & Mar 29, 2016 & $13: 55: 29-16: 27: 52$ & Xinglong $60 / 90 \mathrm{~cm}$ Schmidt & 60 & 126 & 0.22 & Mediocre \\
\hline & Nov 27,2016 & 18:46:02-22:01:22 & Xinglong $60 / 90 \mathrm{~cm}$ Schmidt & 100 & 98 & 0.18 & Golden \\
\hline & Mar 13, 2017 & $13: 01: 32-16: 40: 18$ & Xinglong $60 / 90 \mathrm{~cm}$ Schmidt & 90 & 118 & 0.30 & Mediocre \\
\hline & Mar 26, 2017 & $13: 23: 27-16: 47: 56$ & Xinglong $60 / 90 \mathrm{~cm}$ Schmidt & 100 & 111 & 0.19 & Golden \\
\hline \multirow[t]{2}{*}{ WASP-52 b } & Jan 12,2016 & 10:17:30-11:42:14 & Xinglong $60 \mathrm{~cm}$ & 100 & 49 & 0.32 & Mediocre \\
\hline & Oct 09,2016 & 14:39:28-18:06:37 & Xinglong $60 / 90 \mathrm{~cm}$ Schmidt & 150 & 77 & 0.21 & Mediocre \\
\hline \multirow[t]{5}{*}{ WASP-56 b } & Feb 06, 2016 & $16: 37: 17-21: 21: 43$ & Xinglong $60 \mathrm{~cm}$ & 45 & 333 & 0.13 & Golden \\
\hline & Feb 06, 2016 & $16: 29: 28-20: 34: 20$ & Xinglong $60 / 90 \mathrm{~cm}$ Schmidt & 90 & 141 & 0.19 & Golden \\
\hline & Feb 14, 2017 & 15:44:07-21:09:08 & Xinglong $60 \mathrm{~cm}$ & 15 & 375 & 0.29 & Mediocre \\
\hline & Apr 29,2017 & $12: 31: 36-17: 37: 16$ & Xinglong $60 / 90 \mathrm{~cm}$ Schmidt & 140 & 104 & 0.24 & Mediocre \\
\hline & May 08,2018 & 13:04:20-16:09:08 & Xinglong $60 \mathrm{~cm}$ & 249 & 45 & 0.23 & Mediocre \\
\hline WASP-60 b & Nov 11, 2013 & 10:38:18-15:40:59 & Xinglong $60 / 90 \mathrm{~cm}$ Schmidt & 100 & 125 & 0.12 & Golden \\
\hline \multirow[t]{3}{*}{ WASP-65 b } & Jan 21,2014 & $15: 45: 12-21: 41: 30$ & Xinglong $60 \mathrm{~cm}$ & 60 & 302 & 0.17 & Golden \\
\hline & Jan 21, 2014 & $14: 29: 34-20: 47: 23$ & Xinglong $60 / 90 \mathrm{~cm}$ Schmidt & 80 & 180 & 0.17 & Golden \\
\hline & Jan 10,2016 & 14:09:05-16:17:48 & Xinglong $60 \mathrm{~cm}$ & 30 & 213 & 0.12 & Golden \\
\hline
\end{tabular}

Table 1 continued 
Table 1 (continued)

\begin{tabular}{|c|c|c|c|c|c|c|c|}
\hline Planet & $\begin{array}{l}\text { Date } \\
\text { (UTC) }\end{array}$ & $\begin{array}{l}\text { Time } \\
\text { (UTC) }\end{array}$ & Telescope & $\begin{array}{c}\text { Typical Exposure Time } \\
(\mathrm{s})\end{array}$ & $\mathrm{N}_{\text {data }}$ & PNR & Quality \\
\hline & Nov 03, 2016 & $18: 13: 11-21: 47: 02$ & Xinglong $60 \mathrm{~cm}$ & 20 & 271 & 0.21 & Mediocre \\
\hline & Dec 10, 2016 & 17:09:59-21:47:31 & Xinglong $60 \mathrm{~cm}$ & 20 & 373 & 0.16 & Golden \\
\hline & Jan 23, 2017 & $15: 41: 58-20: 10: 18$ & Xinglong $60 \mathrm{~cm}$ & 22.5 & 323 & 0.16 & Golden \\
\hline & Apr 21,2017 & $11: 42: 30-15: 01: 11$ & Xinglong $60 \mathrm{~cm}$ & 40 & 134 & 0.18 & Golden \\
\hline \multirow[t]{2}{*}{ WASP-80 b } & May 12,2016 & $18: 38: 09-19: 57: 34$ & Xinglong $60 \mathrm{~cm}$ & 25 & 158 & 0.14 & Golden \\
\hline & Sep 12, 2017 & 11:49:35-14:50:21 & Xinglong $60 \mathrm{~cm}$ & 7 & 336 & 0.30 & Mediocre \\
\hline $\mathrm{XO}-2 \mathrm{~b}$ & Feb 17, 2017 & $12: 36: 48-17: 02: 57$ & Xinglong 60/90 cm Schmidt & 40 & 217 & 0.12 & Golden \\
\hline \multirow[t]{2}{*}{$\mathrm{XO}-5 \mathrm{~b}$} & Jan 12,2016 & $17: 58: 54-22: 10: 58$ & Xinglong $60 \mathrm{~cm}$ & 100 & 132 & 0.24 & Mediocre \\
\hline & Jan 29, 2016 & $12: 20: 36-16: 06: 44$ & Xinglong $60 / 90 \mathrm{~cm}$ Schmidt & 150 & 84 & 0.23 & Mediocre \\
\hline
\end{tabular}


WANG ET AL.

Table 2. Photometry of TEMP Targets

\begin{tabular}{ccccc}
\hline \hline Planet & BJD & Relative Flux & Uncertainty & Filter \\
\hline GJ 436 b & 2457456.053648 & 1.0030 & 0.0019 & $\mathrm{R}$ \\
GJ 436 b & 2457456.054238 & 1.0002 & 0.0019 & $\mathrm{R}$ \\
GJ 436 b & 2457456.054840 & 0.9999 & 0.0019 & $\mathrm{R}$ \\
GJ 436 b & 2457456.055430 & 0.9982 & 0.0019 & $\mathrm{R}$ \\
GJ 436 b & 2457456.056032 & 0.9986 & 0.0019 & $\mathrm{R}$ \\
GJ 436 b & 2457456.056622 & 1.0016 & 0.0019 & $\mathrm{R}$ \\
GJ 436 b & 2457456.057224 & 1.0004 & 0.0019 & $\mathrm{R}$ \\
GJ 436 b & 2457456.057815 & 1.0015 & 0.0019 & $\mathrm{R}$ \\
GJ 436 b & 2457456.058416 & 1.0013 & 0.0019 & $\mathrm{R}$ \\
GJ 436 b & 2457456.059018 & 0.9983 & 0.0019 & $\mathrm{R}$ \\
$\ldots$ & $\ldots$ & $\ldots$ & $\ldots$ & $\ldots$ \\
\hline
\end{tabular}

Note: This table is available in its entirety in machine-readable format. A portion of the table is shown here for guidance regarding its form and content. Table 2, 3 and 4 can be downloaded via this link

Table 3. Transit Mid-Times for TEMP Targets

\begin{tabular}{|c|c|c|c|c|}
\hline Planet & Epoch Number & $\begin{array}{c}\mathrm{T}_{0} \\
\left(\mathrm{BJD}_{\mathrm{TDB}}\right)\end{array}$ & $\begin{array}{c}\sigma \\
(\mathrm{s})\end{array}$ & $\begin{array}{l}\mathrm{O}-\mathrm{C} \\
(\mathrm{s})\end{array}$ \\
\hline GJ 436 b & -42 & 2454222.616809 & 65.30 & -7.04 \\
\hline GJ 436 b & -41 & 2454225.260917 & 71.90 & 11.29 \\
\hline GJ $436 \mathrm{~b}$ & -41 & 2454225.261190 & 66.25 & 34.86 \\
\hline GJ $436 \mathrm{~b}$ & -41 & 2454225.261356 & 68.20 & 49.19 \\
\hline GJ $436 \mathrm{~b}$ & -39 & 2454230.548755 & 70.76 & 15.32 \\
\hline GJ 436 b & -36 & 2454238.480865 & 202.79 & 51.88 \\
\hline GJ $436 \mathrm{~b}$ & -34 & 2454243.767054 & 51.72 & -86.56 \\
\hline GJ $436 \mathrm{~b}$ & -33 & 2454246.410962 & 148.03 & -85.43 \\
\hline GJ $436 \mathrm{~b}$ & -33 & 2454246.411611 & 152.83 & -29.37 \\
\hline GJ $436 \mathrm{~b}$ & -31 & 2454251.700230 & 67.85 & 42.17 \\
\hline GJ 436 b & -20 & 2454280.782620 & 21.49 & 2.27 \\
\hline GJ $436 \mathrm{~b}$ & 1181 & 2457456.101184 & 103.67 & 0.52 \\
\hline$\ldots$ & $\ldots$ & $\ldots$ & $\ldots$ & \\
\hline
\end{tabular}

Note: This table is available in its entirety in machine-readable format. A portion of the table is shown here for guidance regarding its form and content. 
New Photometry, New Parameters

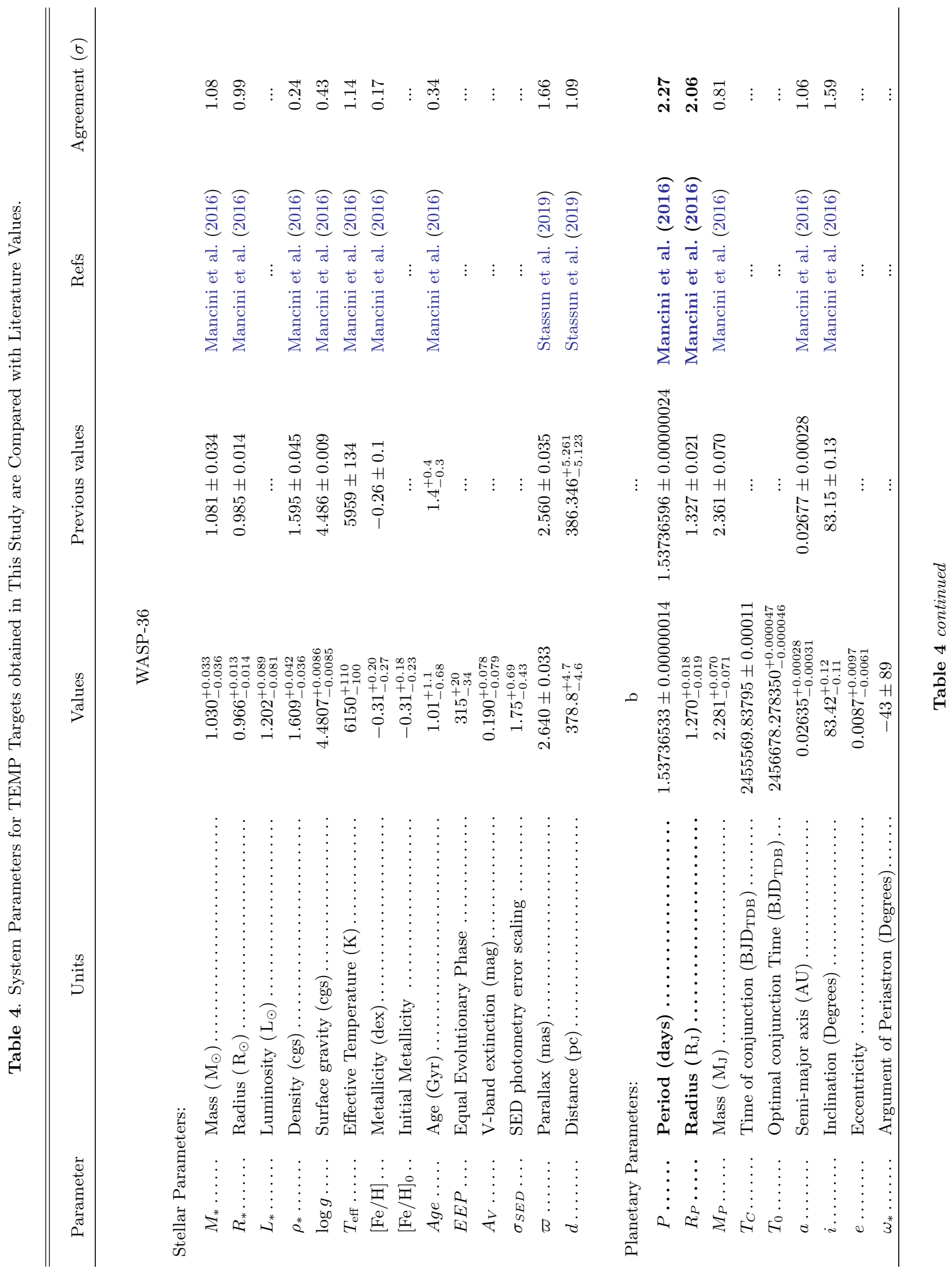




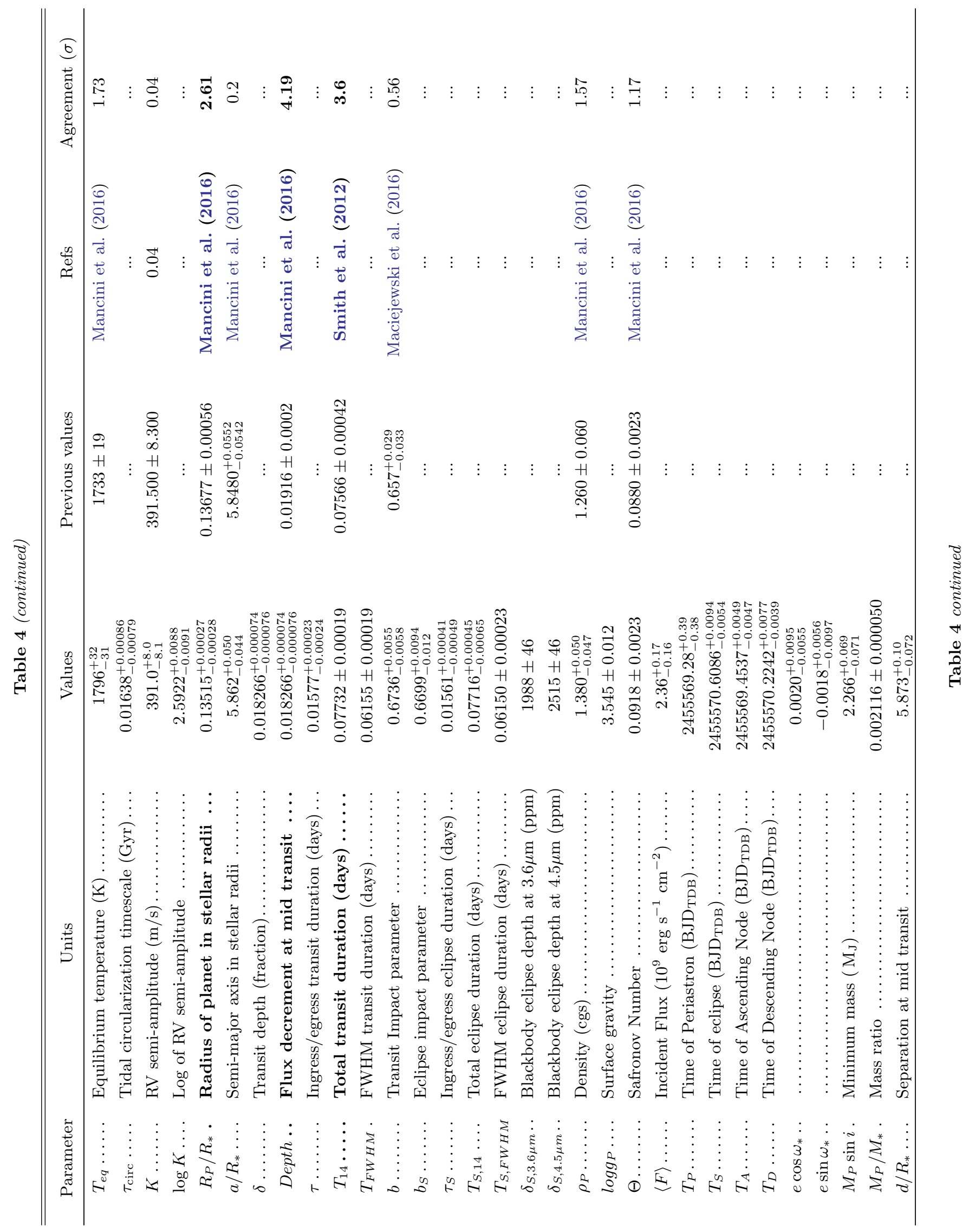




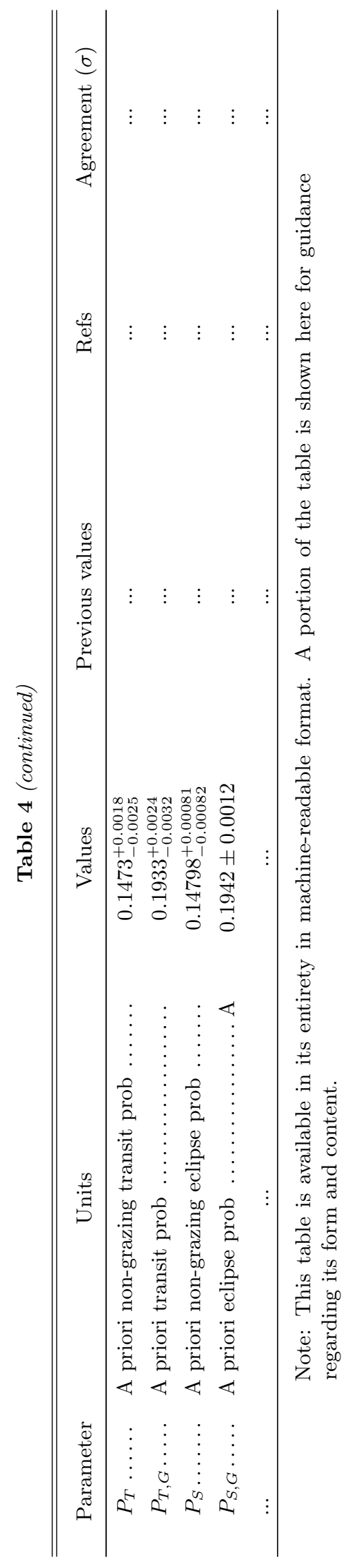


Table 5. Stellar Parameters

\begin{tabular}{|c|c|c|c|c|c|}
\hline Star & $\begin{array}{c}M_{*} \\
\left(\mathrm{M}_{\odot}\right)\end{array}$ & $\begin{array}{c}R_{*} \\
\left(\mathrm{R}_{\odot}\right)\end{array}$ & $\begin{array}{l}\log g \\
(\mathrm{cgs})\end{array}$ & $\begin{array}{l}T_{\text {eff }} \\
(\mathrm{K})\end{array}$ & {$[\mathrm{Fe} / \mathrm{H}]$} \\
\hline GJ 436 & $0.426_{-0.017}^{+0.027}$ & $0.415_{-0.010}^{+0.014}$ & $4.833_{-0.013}^{+0.013}$ & $3370_{-95}^{+94}$ & $-0.46_{-0.24}^{+0.31}$ \\
\hline HAT-P-12 & $0.719_{-0.016}^{+0.016}$ & $0.7084_{-0.0095}^{+0.0095}$ & $4.594_{-0.013}^{+0.013}$ & $4710_{-49}^{+49}$ & $-0.240_{-0.062}^{+0.055}$ \\
\hline HAT-P-16 & $1.187_{-0.065}^{+0.010}$ & $1.217_{-0.022}^{+0.022}$ & $4.342_{-0.027}^{+0.024}$ & $6196_{-71}^{+59}$ & $0.11_{-0.11}^{+0.15}$ \\
\hline HAT-P-18 & $0.750_{-0.014}^{+0.015}$ & $0.7202_{-0.010}^{+0.0095}$ & $4.599_{-0.013}^{+0.013}$ & $4835_{-35}^{+39}$ & $0.044_{-0.051}^{+0.060}$ \\
\hline HAT-P-19 & $0.863_{-0.025}^{+0.029}$ & $0.851_{-0.013}^{+0.013}$ & $4.514_{-0.017}^{+0.019}$ & $5049_{-65}^{+42}$ & $0.283_{-0.079}^{+0.081}$ \\
\hline HAT-P-20 & $0.764_{-0.051}^{+0.042}$ & $0.685_{-0.021}^{+0.016}$ & $4.650_{-0.018}^{+0.018}$ & $4622_{-49}^{+57}$ & $0.23_{-0.24}^{+0.25}$ \\
\hline HAT-P-22 & $1.051_{-0.050}^{+0.045}$ & $0.990_{-0.018}^{+0.018}$ & $4.4687_{-0.013}^{+0.0096}$ & $5426_{-52}^{+55}$ & $0.325_{-0.081}^{+0.084}$ \\
\hline HAT-P-24 & $1.228_{-0.036}^{+0.035}$ & $1.372_{-0.018}^{+0.018}$ & $4.252_{-0.017}^{+0.017}$ & $6488_{-68}^{+70}$ & $-0.056_{-0.054}^{+0.050}$ \\
\hline HAT-P-27 & $0.907_{-0.036}^{+0.040}$ & $0.896_{-0.015}^{+0.015}$ & $4.491_{-0.0}^{+0.0}$ & $5162_{-41}^{+41}$ & $0.210_{-0.097}^{+0.097}$ \\
\hline HAT-P-3 & $0.960_{-0.034}^{+0.031}$ & $0.8627_{-0.012}^{+0.0090}$ & $4.550_{-0.0}^{+0.0}$ & $5133_{-31}^{+36}$ & $0.332_{-0.073}^{+0.074}$ \\
\hline HAT-P-30 & $1.314_{-0.056}^{+0.053}$ & $1.381_{-0.022}^{+0.020}$ & $4.277_{-0}^{+0}$ & $6216_{-48}^{+56}$ & $0.155_{-0.079}^{+0.077}$ \\
\hline HAT-P-37 & $0.953_{-0.040}^{+0.035}$ & $0.8635_{-0.0070}^{+0.0072}$ & $4.545_{-0}^{+0}$ & $5479_{-52}^{+37}$ & $0.22_{-0.12}^{+0.12}$ \\
\hline HAT-P-39 & $1.414_{-0.039}^{+0.039}$ & $1.723_{-0.028}^{+0.028}$ & $4.116_{-0}^{+0}$ & $6412_{-58}^{+56}$ & $0.118_{-0.066}^{+0.074}$ \\
\hline HAT-P-4 & $1.260_{-0.042}^{+0.043}$ & $1.573_{-0 .}^{+0.0}$ & $4.145_{-0}^{+0}$ & $5927_{-47}^{+57}$ & $0.258_{-0 .}^{+0 .(}$ \\
\hline HAT-P-42 & $1.145_{-0.052}^{+0.052}$ & $1.27_{-0.11}^{+0.11}$ & $4.288_{-0}^{+0}$ & $5773_{-40}^{+45}$ & $0.312_{-0.074}^{+0.072}$ \\
\hline HAT-P-43 & $1.042_{-0.035}^{+0.036}$ & $1.076_{-0.025}^{+0.025}$ & $4.392_{-0}^{+0 .}$ & $5656_{-29}^{+37}$ & $0.267_{-0.064}^{+0.061}$ \\
\hline HAT-P-5 & $1.056_{-0.067}^{+0.067}$ & $1.085_{-0.0}^{+0.0}$ & $4.391_{-0}^{+0}$ & $5844_{-52}^{+54}$ & $0.14_{-0.15}^{+0.18}$ \\
\hline HAT-P-52 & $0.891_{-0 .}^{+0 .}$ & $0.886_{-0}^{+0}$ & $4.492_{-0}^{+0}$ & $5144_{-48}^{+47}$ & $0.319_{-0.078}^{+0.076}$ \\
\hline HAT-P-53 & $1.078_{-0.037}^{+0.036}$ & $1.127_{-0}^{+0}$ & $4.367_{-0}^{+0}$ & $5942_{-40}^{+36}$ & $-0.001_{-0.064}^{+0.077}$ \\
\hline HAT-P-54 & $0.706_{-0.025}^{+0.027}$ & $0.6703_{-0.00}^{+0.00}$ & $4.635_{-0}^{+0}$ & $4369_{-71}^{+62}$ & $0.157_{-0 .}^{+0}$ \\
\hline HAT-P-56 & $1.319_{-0.033}^{+0.033}$ & $1.429_{-0.0}^{+0.0}$ & $4.248_{-0}^{+0}$ & $6564_{-46}^{+47}$ & $0.11_{-0.10}^{+0.14}$ \\
\hline HAT-P-8 & $1.270_{-0.030}^{+0.030}$ & $1.491_{-0.0}^{+0.0}$ & $4.1956_{-0.013}^{+0.0095}$ & $6410_{-140}^{+140}$ & $-0.018_{-0.056}^{+0.072}$ \\
\hline KELT-3 & $1.301_{-0.046}^{+0.046}$ & $1.583_{-0.036}^{+0.036}$ & $4.153_{-0}^{+0}$ & $6306_{-35}^{+36}$ & $0.030_{-0.066}^{+0.072}$ \\
\hline Qatar-4 & $0.856_{-0.030}^{+0.029}$ & $0.800_{-0.014}^{+0.015}$ & $4.565_{-0 .}^{+0 .}$ & $5174_{-35}^{+33}$ & $0.095_{-0.088}^{+0.076}$ \\
\hline TrES-1 & $0.886_{-0.022}^{+0.021}$ & $0.8200_{-0.0078}^{+0.0079}$ & $4.5577_{-0.0}^{+0.0}$ & $5316_{-42}^{+43}$ & $0.017_{-0.041}^{+0.045}$ \\
\hline WASP-104 & $1.003_{-0.032}^{+0.030}$ & $0.9277_{-0.0085}^{+0.0089}$ & $4.504_{-0}^{+0}$ & $5392_{-73}^{+69}$ & $0.348_{-0.076}^{+0.076}$ \\
\hline WASP-24 & $1.177_{-0.025}^{+0.025}$ & $1.312_{-0.020}^{+0.019}$ & $4.273_{-0}^{+0 .}$ & $6489_{-74}^{+89}$ & $-0.37_{-0.18}^{+0.12}$ \\
\hline WASP-28 & $1.082_{-0.071}^{+0.068}$ & $1.132_{-0.018}^{+0.019}$ & $4.365_{-0}^{+0}$ & $5976_{-60}^{+54}$ & $0.06_{-0.12}^{+0.17}$ \\
\hline WASP-36 & $1.030_{-0.036}^{+0.033}$ & $0.966_{-0.014}^{+0.013}$ & $4.4807_{-0.0085}^{+0.0086}$ & $6150_{-100}^{+110}$ & $-0.31_{-0.27}^{+0.20}$ \\
\hline WASP-37 & $0.926_{-0.034}^{+0.036}$ & $1.071_{-0 .}^{+0 .}$ & $4.346_{-0 .}^{+0 .}$ & $\begin{array}{l}5795_{-64}^{+69} \\
\end{array}$ & $-0.098_{-0.060}^{+0.050}$ \\
\hline WASP-38 & $1.200_{-0.029}^{+0.029}$ & $1.368_{-0.014}^{+0.014}$ & $4.2450_{-0.0083}^{+0.0081}$ & $6168_{-58}^{+57}$ & $\begin{array}{r}-0.060 \\
-0.001_{-0.064}^{+0.065}\end{array}$ \\
\hline WASP-43 & $0.723_{-0.031}^{+0.028}$ & $0.6747_{-0.0097}^{+0.0086}$ & $4.639_{-0 .}^{+0 .}$ & $4286_{-40}^{+34}$ & $0.28_{-0.1}^{+0.064}$ \\
\hline WASP-52 & $0.886_{-0.043}^{+0.039}$ & $0.825_{-0.0}^{+0.0}$ & $4.553_{-0}^{+0}$ & $5070_{-40}^{+35}$ & $0.37_{-0}^{+0}$ \\
\hline WASP-56 & $1.092_{-0.023}^{+0.023}$ & $1.129_{-0.0}^{+0.0}$ & $4.371_{-0}^{+0}$. & $5646_{-48}^{+49}$ & $0.160_{-0.057}^{+0.057}$ \\
\hline WASP-60 & $1.082_{-0.031}^{+0.032}$ & $1.384_{-0.0}^{+0.0}$ & $4.190_{-0.027}^{+0.026}$ & $5926_{-76}^{+77}$ & $0.023_{-0.055}^{+0.067}$ \\
\hline WASP-65 & $1.098_{-0.052}^{+0.031}$ & $1.056_{-0.018}^{+0.017}$ & $4.430_{-0.015}^{+0.0216}$ & $5749_{-48}^{+45}$ & $0.447_{-0.13}^{+0.086}$ \\
\hline WASP-80 & $0.614_{-0.012}^{+0.052}$ & $0.6023_{-0.0052}^{+0.018}$ & $4.6672_{-0.011}^{+0.015}$ & $4158_{-49}^{+41}$ & $-0.011_{-0.017}^{+0.034}$ \\
\hline $\mathrm{XO}-2$ & $0.967_{-0.028}^{+0.012}$ & $1.004_{-0.013}^{+0.0052}$ & $4.420_{-0.016}^{+0.011}$ & $5360_{-50}^{+50}$ & $0.436_{-0.046}^{+0.043}$ \\
\hline $\mathrm{XO}-5$ & $1.053_{-0.062}^{+0.064}$ & $1.065_{-0.026}^{+0.026}$ & $4.406_{-0.029}^{+0.029}$ & $5515_{-68}^{+69}$ & $0.447_{-0.12}^{+0.079}$ \\
\hline
\end{tabular}


-

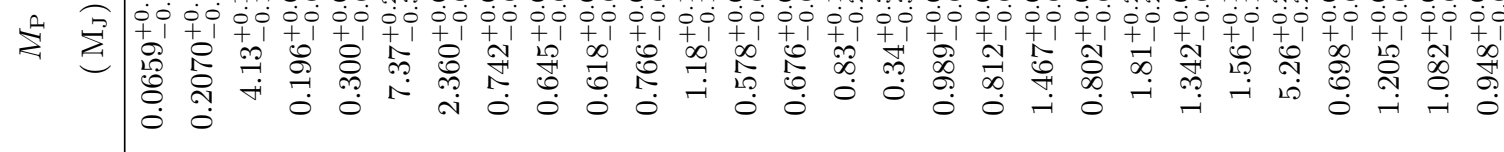

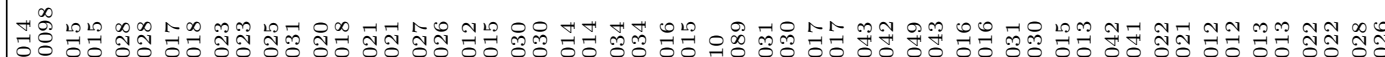
Q

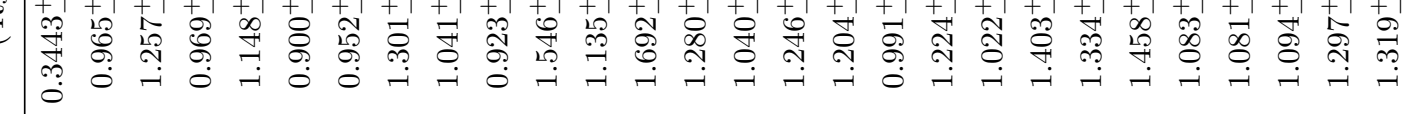

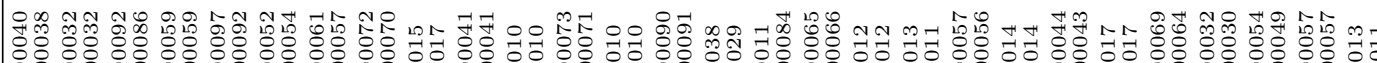

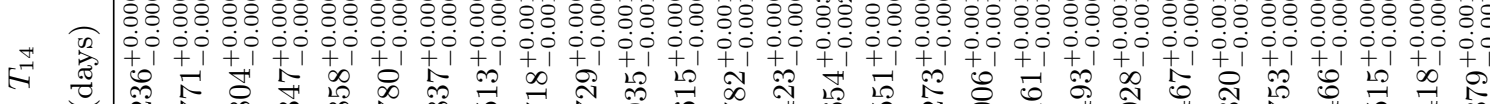

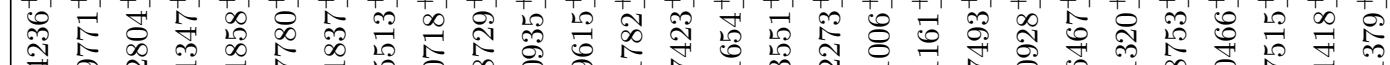

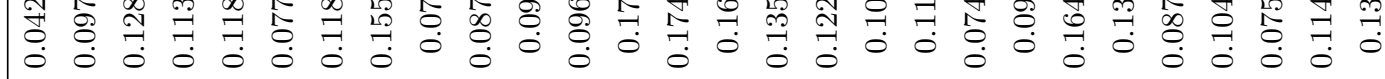

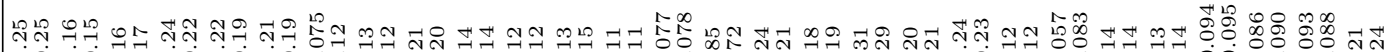

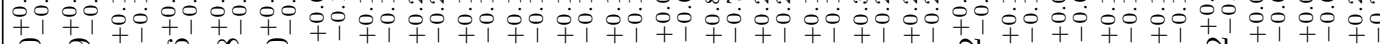

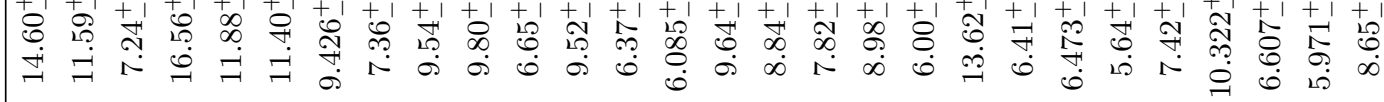

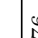

$\stackrel{\underbrace{*}}{\stackrel{2}{\infty}}$

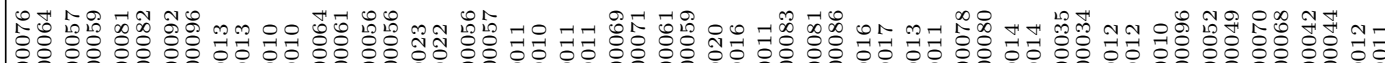

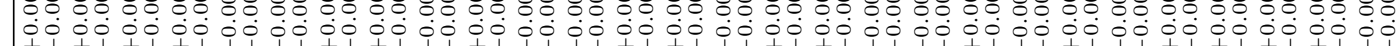

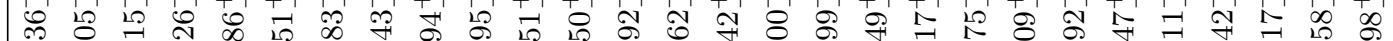

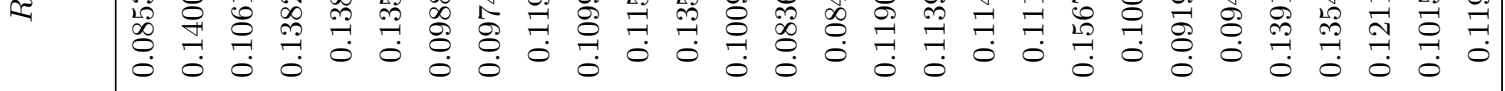

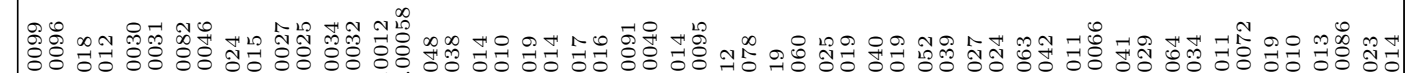
$\begin{array}{llllllll}0 & 0\end{array}$

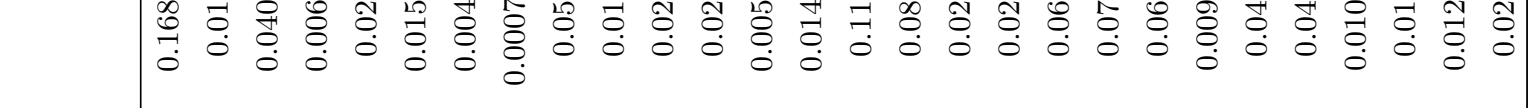

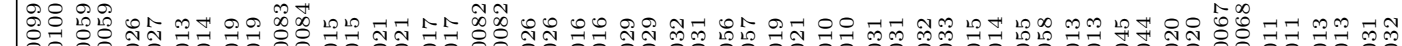

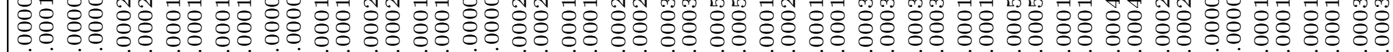
ol

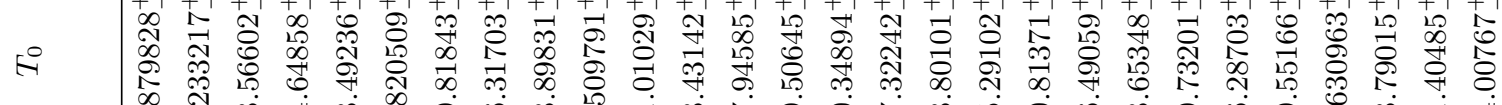

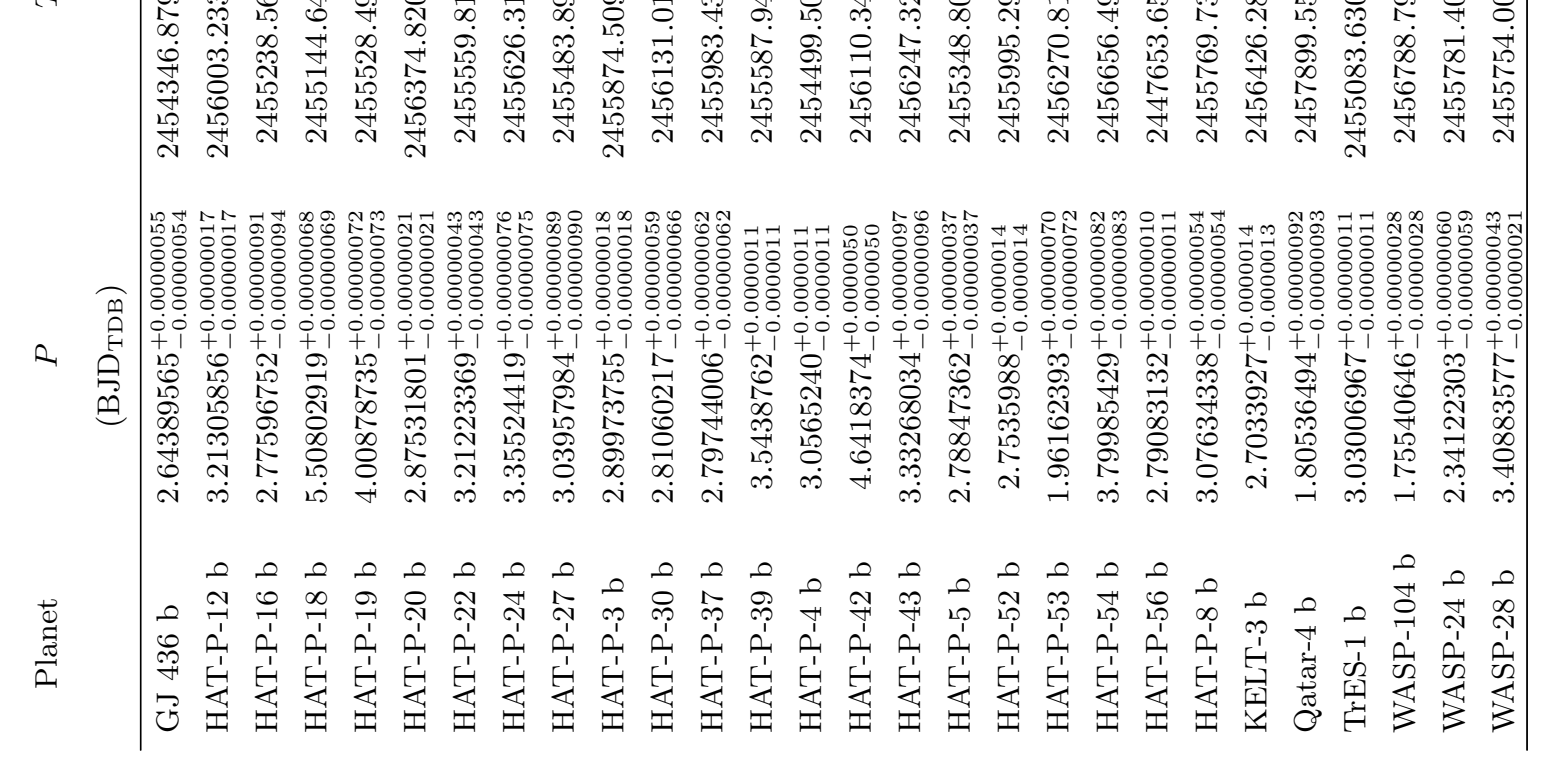




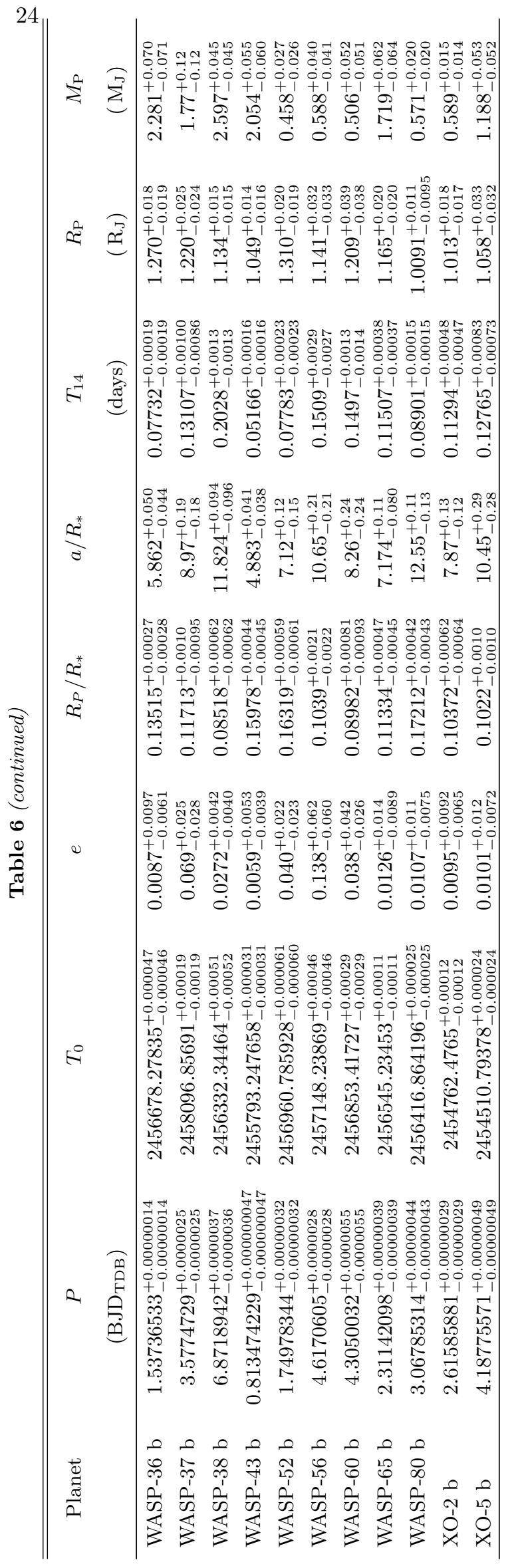

WANG ET AL. 


\section{ACKNOWLEDGMENTS}

We thank Jason Eastman for his useful discussions. We also would like to thank Metrics for advice on figure designs that has led to a considerable improvement of the manuscript. S.W. acknowledges support from Indiana University, Yale University, and the Heising-Simons Foundation. M.R. is supported by the National Science Foundation Graduate Research Fellowship Program under Grant Number DGE-1752134. TCH acknowledges financial support from the National Research Foundation (NRF; No. 2019R1I1A1A01059609). M.B. and Y.W. thank the support of the National Natural Science Foundation of China (Grant No. 12073092). Y.W. thanks the fellowship of the China Postdoctoral Science Foundation (Grant No. 2020M672936). H.Z. thanks the support of the National Natural Science Foundation of China (Grant No. 12073010). This work is supported by the National Natural Science Foundation of China (NSFC) (11803055), the Joint Research Fund in Astronomy (U1731125, U1731243, U1931132) under cooperative agreement between the NSFC and Chinese Academy of Sciences (CAS), the 13th Five-year Informatization Plan of Chinese Academy of Sciences (No. XXH-13514, XXH13503-03-107). This work is supported by Astronomical Big Data Joint Research Center, co-founded by National Astronomical Observatories, Chinese Academy of Sciences and Alibaba Cloud. We acknowledge the support of the staff of the Xinglong 60 $\mathrm{cm}$ telescope. This work was partially supported by the Open Project Program of the Key Laboratory of Optical Astronomy, National Astronomical Observatories, Chinese Academy of Sciences. 


\section{REFERENCES}

Addison, B. C., Wang, S., Johnson, M. C., et al. 2018, AJ, 156, 197

Addison, B. C., Wright, D. J., Nicholson, B. A., et al. 2020, MNRAS, 502, 3704

Agol, E. \& Deck, K. 2016, ApJ, 818, 177

Agol, E., Steffen, J., Sari, R., \& Clarkson, W. 2005, MNRAS, 359, 567

Albrecht, S., Winn, J. N., Johnson, J. A., et al. 2012, ApJ, 757,18

Alonso, R., Brown, T. M., Torres, G., et al. 2004, ApJL, 613, L153

Alsubai, K. A., Parley, N. R., Bramich, D. M., et al. 2013, AcA, 63, 465

Auvergne, M., Bodin, P., Boisnard, L., et al. 2009, A\&A, 506, 411

Bakos, G. Á., Noyes, R. W., Kovács, G., et al. 2004, PASP, 116, 266

Bakos, G. Á., Csubry, Z., Penev, K., et al. 2013, PASP, 125,154

Batygin, K., Bodenheimer, P. H., \& Laughlin, G. P. 2016, ApJ, 829, 114

Becker, J. C., Vanderburg, A., Adams, F. C., et al. 2015, ApJL, 812, L18

Bertin, E., Arnouts, S. 1996, A\&AS, 117, 393B

Bianchi, L., Herald, J., Efremova, B., et al. 2011, Ap\&SS, 335,161

Boley, A. C., Granados Contreras, A. P., \& Gladman, B. 2016, ApJL, 817, L17

Borucki, W. J., Koch, D., Basri, G., et al. 2010, Science, 327,977

Borucki, W. J., Koch, D., Jenkins, J., et al. 2009, Science, 325,709

Bouma, L. G., Winn, J. N., Baxter, C., et al. 2019, AJ, 157, 217

Brahm, R., Nielsen, L. D., Wittenmyer, R. A., et al. 2020, AJ, 160, 235

Carter, J. A., \& Winn, J. N. 2010, ApJ, 709, 1219

Cañas, C. I., Wang, S., Mahadevan, S., et al. 2019, ApJL, 870, L17

Cañas, C. I., Stefansson, G., Monson, A. J., et al. 2019, ApJL, 877

Charbonneau, D., Allen, L. E., Megeath, S. T., et al. 2005, ApJ, 626, 523

Charbonneau, D., Brown, T. M., Noyes, R. W., \& Gilliland, R. L. 2002, ApJ, 568, 377

Cincotta, P. M., \& Simó, C. 1999, Celestial Mechanics and Dynamical Astronomy, 73, 195

Cincotta, P. M., \& Simó, C. 2000, A\&AS, 147, 205
Cincotta, P. M., Giordano, C. M., \& Simó, C. 2003, Physica D Nonlinear Phenomena, 182, 151

Claret, A. 2018, A\&A, 618, A20

Cortés-Zuleta, P., Rojo, P., Wang, S., et al. 2020, A\&A, 636, A98

Cutri, R. M., Et, A. 2013, Vizier Online Data Catalog, II $/ 328$

Cutri, R. M., Skrutskie, M. F., van Dyk, S., et al. 2003, The IRSA 2MASS All-Sky Point Source Catalog, NASA/IPAC Infrared Science Archive.

Davis, A. B., Wang, S., Jones, M., et al. 2020, AJ, 160, 229

Deming, D., \& Seager, S. 2017, arXiv:1701.00493

Deming, D., Seager, S., Richardson, L. J., \& Harrington, J. 2005, Nature, 434, 740

Eastman, J., Gaudi, B. S., Agol, E. 2013, PASP, 125, 83

Eastman, J., Siverd, R., \& Gaudi, B. S. 2010, PASP, 122, 935

Eastman, J., Rodriguez, J. E., Agol, E., et al. 2019, Arxiv E-prints, arXiv:1907.09480

Eastman, J. 2017, Astrophysics Source Code Library. ascl:1710.003

Fulton, B., Shporer, A., Winn, j.. et al. 2011, AJ, 142, 84

Gaia, C., Brown, A. G. A., Vallenari, A., et al. 2016, A\&A, 595, A2

Gaia Collaboration, Brown, A. G. A., Vallenari, A., et al. 2018, A\&A, 616, A1

Gardner, J. P., Mather, J. C., Clampin, M., et al. 2006, SSRv, 123, 485

Gelman, A. \& Rubin, D. B. 1992, Statistical Science, 7, 457

Gillon, M., Triaud, A. H. M. J., Fortney, J. J., et al. 2012, A\&A, 542, A4

Greiss, S., Steeghs, D., Gänsicke, B. T., et al. 2012, AJ, 144,24

Henden, A. A., Templeton, M., Terrell, D., et al. 2016, Vizier Online Data Catalog, II/336

Hinse, T. C., Christou, A. A., Alvarellos, J. L. A., \& Go'zdziewski, K. 2010, MNRAS, 404, 837

Holman, M. J., \& Murray, N. W. 2005, Science, 307, 1288

Holman, M. J., Winn, J. N., Latham, D. W., et al. 2006, ApJ, 652, 1715

Holt, J. R. 1893, Astronomy and Astro-Physics (formerly The Sidereal Messenger), 12, 646

Howell, S. B., Sobeck, C., Haas, M., et al. 2014, PASP, 126, 398

Huang, C. X., Petrovich, C., \& Deibert, E. 2017, AJ, 153, 210

Huang, C. X., Quinn, S. N., Vanderburg, A., et al. 2020, ApJL, 892, L7 
Hébrard, G., Bouchy, F., Pont, F., et al. 2008, A\&A, 488, 763

Hébrard, G., Díaz, R. F., Correia, A. C. M., et al. 2020, A\&A, 640, A32

Høg, E., Fabricius, C., Makarov, V. V., et al. 2000, A\&A, 355, L27

Irwin, J., Charbonneau, D., Nutzman, P., \& Falco, E. 2009, Transiting Planets, 253, 37

Jones, M. I., Brahm, R., Espinoza, N., et al. 2019, A\&A, 625, A16

Jordán, A., Brahm, R., Espinoza, N., et al. 2020, AJ, 159, 145

Knutson, H. A., Lewis, N., Fortney, J. J., et al. 2012, ApJ, 754,22

Kreidberg, L., Bean, J. L., Désert, J.-M., et al. 2014, Nature, 505, 69

Laughlin G., Crismani M., Adams F. C., 2011, 729(1), L7

Lucy, L. B. \& Sweeney, M. A. 1971, AJ, 76, 544

Maciejewski, G., Dimitrov, D., Mancini, L., et al. 2016, AcA, 66, 55

Macqueen, J. B. 1967, 281-297, "Some Methods for Classification and Analysis of Multivariate

Observations," In: Proceedings of 5th Berkeley Symposium on Mathematical Statistics and Probability, University of California Press, Berkeley

Mallonn, M., Von, E. C., Herrero, E., et al. 2019, A\&A, 622, A 81

Mancini, L., Kemmer, J., Southworth, J., et al. 2016, MNRAS, 459, 1393

McCullough, P. R., Stys, J. E., Valenti, J. A., et al. 2005, PASP, 117, 783

McLaughlin, D. B. 1924, ApJ, 60, 22

Mermilliod, J. -C. 1994, Vizier Online Data Catalog, II/193

Millholland, S., Wang, S., \& Laughlin, G. 2016, ApJL, 823, L7

Montalto, M., Gregorio, J., Boué, G., et al. 2012, MNRAS, 427, 2757. doi:10.1111/j.1365-2966.2012.21926.x

Nascimbeni, V., Piotto, G., Bedin, L. R., et al. 2011, A\&A, 527, A85

Neuhäuser, R., Errmann, R., Berndt, A., et al. 2011, Astronomische Nachrichten, 332, 547

Paunzen, E. 2015, A\&A, 580, A23

Penev, K., Bouma, L. G., Winn, J. N., \& Hartman, J. D. 2018, AJ, 155, 165

Pepper, J., Pogge, R. W., DePoy, D. L., et al. 2007, PASP, 119, 923

Pollacco, D. L., Skillen, I., Collier Cameron, A., et al. 2006, PASP, 118, 1407
Press W. H., Flannery B. P., Teukolsky S. A., Vetterling W. T., 1992, Numerical Recipes in Fortran 77, 2nd edn. Cambridge Univ. Press, Cambridge

Pál, A., \& Kocsis, B. 2008, MNRAS, 389, 191

Queloz, D., Eggenberger, A., Mayor, M., et al. 2000, A\&A, 359, L13

Rein, H. \& Liu, S.-F. 2012, A\&A, 537, A128

Rein, H. \& Spiegel, D. S. 2015, MNRAS, 446, 1424

Ricker, G. R., Winn, J. N., Vanderspek, R., et al. 2015, Journal of Astronomical Telescopes, Instruments, and Systems, 1, 014003

Rossiter, R. A. 1924, ApJ, 60, 15

Schlafly, E. F., Finkbeiner, D. P. 2011, ApJ, 737, 103

Schlaufman, K. C., \& Winn, J. N. 2013, ApJ, 772, 143

Schlesinger, F. 1910, Publications of the Allegheny

Observatory of the University of Pittsburgh, 1, 123

Seager, S., \& Hui, L. 2002, ApJ, 574, 1004

Seager, S. \& Mallén-Ornelas, G. 2003, ApJ, 585, 1038

Smith, A. M. S., Anderson, D. R., Collier, C. A., et al. 2012, AJ, 143, 81

Southworth, J., Maxted, P. F. L., Smalley, B. 2004, MNRAS, 349, 547

Southworth, J., Maxted, P. F. L., Smalley, B. 2004, MNRAS, 351, 1277

Southworth, J., Hinse, T. C., Jørgensen, U. G., et al. 2009, MNRAS, 396, 1023

Stassun, K. G., \& Torres, G. 2018, ApJ, 862, 61

Stassun, K. G., Oelkers, R. J., Paegert, M., et al. 2019, AJ, 158,138

Steffen, J. H., Ragozzine, D., Fabrycky, D. C., et al. 2012, Proceedings of the National Academy of Sciences of the United States of America, 109, 7982

Talens, G. J. J., Spronck, J. F. P., Lesage, A.-L., et al. 2017, A\&A, 601, A11

Ter Braak C. J. F. 2006, Stat. Comput., 16, 239

Thorngren, D. P., Fortney, J. J., et al. 2018, AJ, 155, 214 T

Udalski, A., Paczynski, B., Zebrun, K., et al. 2002, AcA, 52,1

Wang, S., Addison, B., Fischer, D. A., et al. 2018a, AJ, 155,70

Wang, S., Jones, M., Shporer, A., et al. 2019, AJ, 157, 51

Wang, S., Wang, X.-Y., Wang, Y.-H., et al. 2018b, AJ, 156, 181

Wang, S., Wu, D.-H., Barclay, T., et al. 2017, arXiv:1704.04290

Wang, S., Zhang, H., Zhou, J.-L., et al. 2014, ApJS, 211, 26

Wang, X.-Y., Wang, S., Hinse, T. C., et al. 2018, PASP, 130, 064401

Wang, Y.-H., Wang, S., Liu, H.-G., et al. 2017, AJ, 154, 49 
Wang, Y.-H., Wang, S., Hinse, T. C., et al. 2019, AJ, 157, 82

Wheatley, P. J., West, R. G., Goad, M. R., et al. 2018, MNRAS, 475, 4476

Winn, J. N., \& Fabrycky, D. C. 2015, ARA\&A, 53, 409

Wittenmyer, R. A., Clark, J. T., Zhao, J., et al. 2019, MNRAS, 484, 5859

Wittenmyer, R. A., Wang, S., Horner, J., et al. 2013, ApJS, 208, 2
Wu, D.-H., Wang, S., Zhou, J.-L., et al. 2018, AJ, 156, 96 Zacharias, N., Finch, C. T., Girard, T. M., et al. 2012, Vizier Online Data Catalog, I/322A

Zhou, G., Rodriguez, J. E., Collins, K. A., et al. 2016, AJ, 152,136

Zhou, X., Chen, J., Xu, W., et al. 1999, PASP, 111, 909

Zhou, X., Jiang, Z., Xue, S., et al. 2001, ChJA\&A, 1,372 